\title{
NEW POINTS OF VIEW IN KNOT THEORY
}

\author{
JOAN S. BIRMAN
}

\section{INTRODUCTION}

In this article we shall give an account of certain developments in knot theory which followed upon the discovery of the Jones polynomial [Jo3] in 1984. The focus of our account will be recent glimmerings of understanding of the topological meaning of the new invariants. A second theme will be the central role that braid theory has played in the subject. A third will be the unifying principles provided by representations of simple Lie algebras and their universal enveloping algebras. These choices in emphasis are our own. They represent, at best, particular aspects of the far-reaching ramifications that followed the discovery of the Jones polynomial.

We begin in $\S 1$ by discussing the topological underpinnings of that most famous of the classical knot invariants-the Alexander polynomial. It will serve as a model for the sort of thing one would like to see for the Jones polynomial. Alexander's 1928 paper ends with a hint of things to come, in the form of a crossing-change formula for his polynomial, and in $\S 2$ we discuss how related formulas made their appearances in connection with the Jones polynomial and eventually led to the discovery of other, more general knot and link polynomials. A more systematic description of these "generalized Jones invariants" is given in $\S 3$, via the theory of $\mathbf{R}$-matrices. That is where braids enter the picture, because every generalized Jones invariant is obtained from a trace function on an " $R$ matrix representation" of the family of braid groups $\left\{B_{n} ; n=1,2,3, \ldots\right\}$. The mechanism for finding R-matrix representations of $B_{n}$ is the theory of quantum groups. For this reason, the collection of knot and link invariants that they determine have been called quantum group invariants. We shall refer to them here as either quantum group invariants or generalized Jones invariants, interchangeably. While the theory of R-matrices and their construction via quantum groups gives a coherent and uniform description of the entire class of invariants, the underlying ideas will be seen to be essentially combinatorial in nature. Thus, by the end of $\S 3$ the reader should begin to understand how it could happen that in 1990 topologists had a fairly coherent framework for constructing vast new families of knot and link invariants, possibly even enough to classify unoriented knot and link types, without having the slightest clue to the underlying topology.

Received by the editors September 30, 1992.

1991 Mathematics Subject Classification. Primary 57M25; Secondary 57N99.

Key words and phrases. Knots, links, knot polynomials, knot groups, Vassiliev invariants, Rmatrices, quantum groups.

This research was supported in part by NSF Grant DMS-91-06584 and by the US-Israel Binational Science Foundation Grant 89-00302-2.

This manuscript is an expanded version of an AMS-MAA Invited Address, given on January 8 , 1992, at the Joint Winter Meeting of the AMS and the MAA in Baltimore, Maryland. 
In $\S 4$ we introduce an entirely new collection of invariants, which arose out of techniques pioneered by Arnold in singularity theory (see the introduction to [Arn1, Arn2]). The new invariants will be seen to have a solid basis in a very interesting new topology, where one studies not a single knot, but a space of all knots. This point of view was carried out successfully for the case of knots by Vassiliev [V]. (Remark. For simplicity, we restrict our attention in this part of the review to knots. The theory is, at this writing, less well developed for links.) Vassiliev's knot invariants are rational numbers. They lie in vector spaces $V_{i}$ of dimension $d_{i}, i=1,2,3, \ldots$, with invariants in $V_{i}$ having "order" $i$. On the other hand, quantum group invariants are Laurent polynomials over $Z$, in a single variable $q$. The relationship between them is as follows:

Theorem 1. Let $\mathscr{F}_{q}(\mathbf{K})$ be a quantum group invariant for a knot type $\mathbf{K}$. Let

$$
\mathscr{P}_{x}(\mathbf{K})=\sum_{i=0}^{\inf } u_{i}(\mathbf{K}) x^{i}
$$

be the power series over the rational numbers $Q$ obtained from $\mathscr{J}_{q}(\mathbf{K})$ by setting $q=e^{x}$ and expanding the powers of $e^{x}$ in their Taylor series. Then the coefficient $u_{i}(\mathbf{K})$ of $x^{i}$ is 1 if $i=0$ and a Vassiliev invariant of order $i$ for each $i \geq 1$.

Thus Vassiliev's topology of the space of all knots suggests the topological underpinnings we seek for the quantum group knot invariants.

A crucial idea in the statement of Theorem 1 is that one must pass to the power series representation of the knot polynomials before one can understand the situation. This was first explained to the author and Lin by Bar Natan. Theorem 1 was first proved for special cases in [BL] and then generalized in [Li1]. In $\S \S 5-7$ we describe a set of ideas which will be seen to lead to a new and very simple proof of Theorem 1. First, in $\S 5$, we review how Vassiliev's invariants, like the Jones polynomial, the HOMFLY and Kauffman polynomials [FHL, Kau1], and the $\mathbf{G}_{2}$ polynomial [Ku] can be described by axioms and initial data. (Actually, all of the quantum group invariants admit such a description, but the axioms can be very complicated; so such a description would probably not be enlightening). In $\S 6$ we introduce braids into the Vassiliev setting, via a new type of object, the monoid of singular braids. Remarkably, this monoid will be seen to map homomorphically (we conjecture isomorphically) into the group algebra of the braid group, implying that it is as fundamental an object in mathematics as the braid group itself. This allows us to extend every R-matrix representation of the braid group to the singular braid monoid. In $\S 7$ we use R-matrices and singular braids to prove Theorem 1 . In $\S 8$ we return to the topology, discussing the beginning of a topological understanding of the quantum group invariants. We then discuss, briefly, a central problem: do we now know enough to classify knots via their algebraic invariants? We will describe some of the evidence which allows us to sharpen that question.

Our goal, throughout this review, is to present the material in the most transparent and nontechnical manner possible in order to help readers who work in other areas to learn as much as possible about the state of the art in knot theory. Thus, when we give "proofs", they will be, at best, sketches of proofs. We hope there will be enough detail so that, say, a diligent graduate student who is motivated to read a little beyond this paper will be able to fill in the gaps. 
Among the many topics which we decided deliberately to exclude from this review for reasons of space, one stands out: it concerns the generalizations of the quantum group invariants and Vassiliev invariants to knots and links in arbitrary 3-manifolds, i.e., the program set forth by Witten in [Wi]. That very general program is inherently more difficult than the special case of knots and links or simply of knots in the 3-sphere. It is an active area of research, with new discoveries made every day. We thought, at first, to discuss, very briefly, the 3-manifold invariants of Reshetikhin and Turaev [RT] and the detailed working out of special cases of those invariants by Kirby and Melvin [KM]; however, we then realized that we could not include such a discussion and ignore Jeffrey's formulas for the Witten invariants of the lens spaces [Je]. Reluctantly, we made the decision to restrict our attention to knots in 3-space, but still, we have given at best a restricted picture. For example, we could not do justice to the topological constructions in [La] and in [Koh1, Koh2] without making this review much longer than we wanted it to be, even though it seems very likely that those constructions are closely related to the central theme of this review. We regret those and other omissions.

\section{AN INTRODUCTION TO KNOTS AND THEIR ALEXANDER INVARIANTS}

We will regard a knot $K$ as the embedded image of an oriented circle $S^{1}$ in oriented 3-space $\mathbb{R}^{3}$ or $S^{3}$. If, instead, we begin with $\mu \geq 2$ copies of $S^{1}$, the image (still called $K$ ), is a link. Its type $\mathbf{K}$ is the topological type of the pair $\left(S^{3}, K\right)$, under homeomorphisms which preserve orientations on both $S^{3}$ and $K$. Knot types do not change if we replace $S^{3}$ by $\mathbb{R}^{3}$, because every homeomorphism of $S^{3}$ is isotopic to one which fixes one point, and that point may be chosen to be the point at infinity.

We may visualize a knot via a diagram, i.e., a projection of $K \subset \mathbb{R}^{3}$ onto a generic $\mathbb{R}^{2} \subset \mathbb{R}^{3}$, where the image is decorated to distinguish overpasses from underpasses, for example, as for highways on a map. Examples are given in Figure 1. The example in Figure 1(a) is the unknot, represented by a round planar circle. Figure 1(b) shows a layered diagram of a knot, i.e., one which has been drawn, using an arbitrary but fixed starting point (which in Figure 1(c) is the tip of the arrow) without the use of an eraser, so that the first passage across a double point in the projection is always an overpass. We leave it to the reader to prove that a layered diagram with $\mu$ components always represents $\mu$ unknotted, unlinked circles. From this simple fact it follows that any diagram of any link may be systematically changed to a diagram for the unlink on the same number of components by finitely many crossing changes.

The diagram in Figure 1(c) was chosen to illustrate the subtleties of knot diagrams. It too represents the unknot, but it is not layered. This example was constructed by Goeritz [Go] in the mid-1930s. At that time it was known that a finite number of repetitions of Reidemeister's three famous moves, depicted (up to obvious symmetries and variations) in Figure 2, suffice to take any one diagram of a knot to any other. Notice that Reidemeister's moves are "local" in the sense that they are restricted to regions of the diagram which contain at most three crossings. If one defines the complexity of a knot diagram to be its crossing number, a natural question is whether there is a set of moves that preserve or reduce complexity and that, applied repeatedly, suffice to reduce an arbitrary diagram of an arbitrary knot to one of minimum crossing number. The 


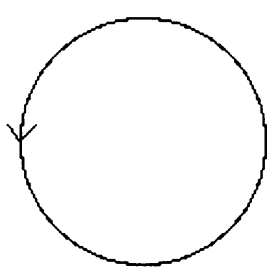

(a)

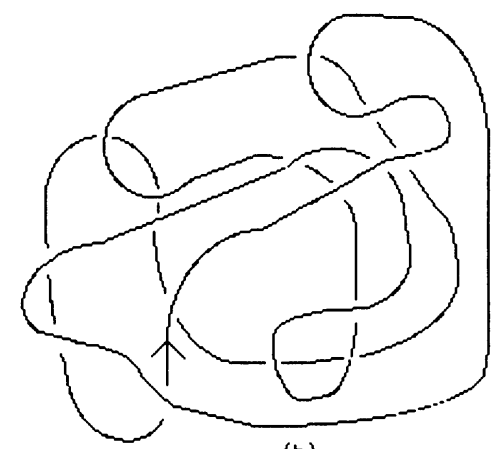

(b)

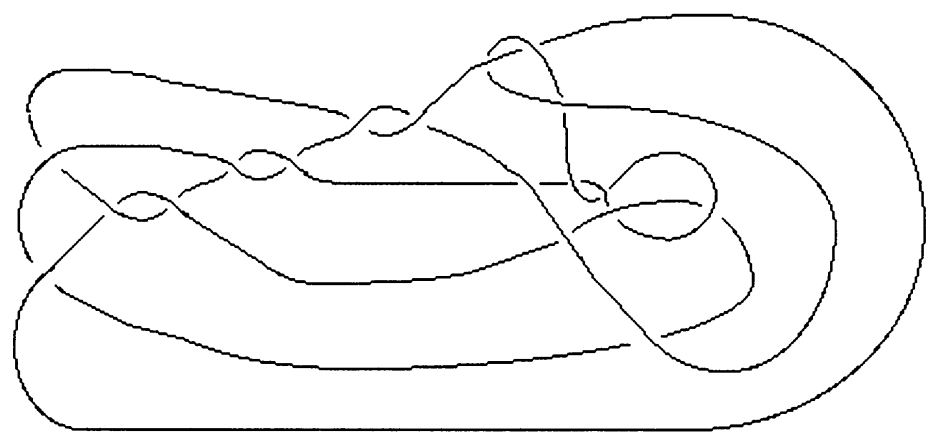

(c)

FIGURE 1. Diagrams of the unknot.

diagram in Figure 1(c) effectively ended that approach to the subject, because the eight moves which had by then been proposed as augmented Reidemeister moves did not suffice to simplify this diagram.

Some insight may be obtained into the question by inspecting the "handle move" of Figure 3. Note that the crossing number in the diagram of Figure 1(c) can be reduced by an appropriate handle move. The region that is labeled $X$ in Figure 3 is arbitrary. The handle move clearly decreases crossing number, but a few moments of thought should convince the reader that if one tries to
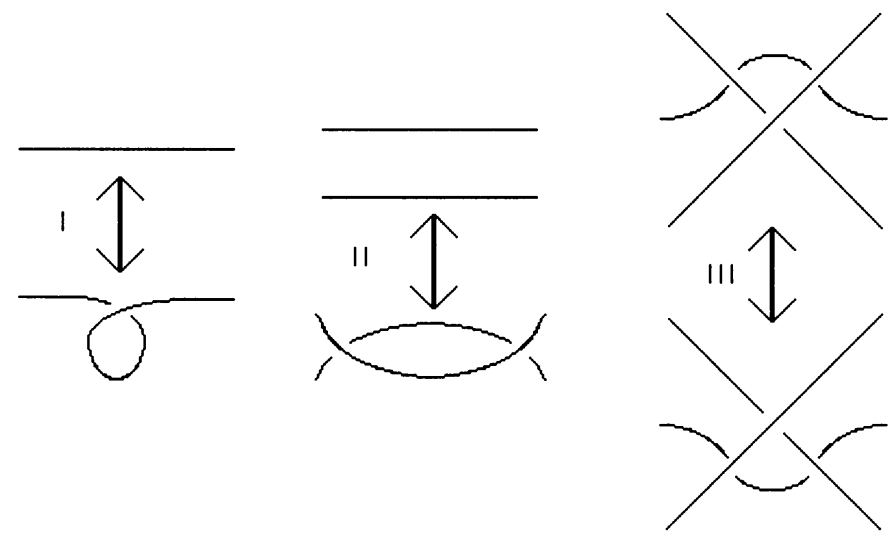

FiguRE 2. Reidemeister moves. 


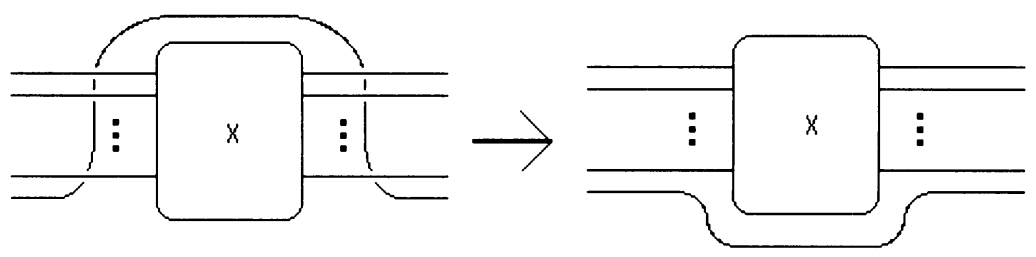

Figure 3. A handle move.

factorize it into a product of Reidemeister moves, for any sufficiently complicated choice of $X$, it will be necessary to use the second Reidemeister move repeatedly in order to create regions in which the third move may be applied. The same sort of reasoning makes it unlikely that any set of local moves suffices to reduce complexity. Indeed, if we knew such a set of moves, we would have the beginning of an algorithm for solving the knot problem, because there are only finitely many knot diagrams with fixed crossing number.

In Figure 4 we have given additional examples, taken from the beginning of a table compiled at the end of the nineteenth century by the physicist Peter Guthrie Tait and coworkers, as part of a systematic effort to classify knots. The knots in the table are listed in order of their crossing number, so that, for example, 76 is the 6 th knot that was discovered with 7 crossings. The part of the table which we have shown includes all prime knots with at most 7 crossings, up to the symmetries defined by reversing the orientation on either $K$ or $S^{3}$. (We have shown the two trefoils, for reasons which will become clear shortly.) The tables, which eventually included all prime knots defined by diagrams with at most 13 crossings, were an ambitious undertaking. (Aside: Yes, it is true. Knots, like integers, have a decomposition into an appropriately defined product of prime knots, and this decomposition is unique up to order.) Their clearly stated goal [Ta] was to uncover the underlying principles of knotting, but to the great disappointment of all concerned they did not even succeed in revealing a single knot type invariant which could be computed from a diagram. Their importance, to this day, is due to the fact that they provide a rich source of examples and convincing evidence of both the beauty and subtlety of the subject.

Beneath each of the examples in Figure 4 we show two famous invariants, namely, the Alexander polynomial $A_{q}(\mathbf{K})$ and the one-variable Jones polynomial $J_{q}(\mathbf{K})$. Both are to be regarded as Laurent polynomials in $q$, the series of numbers representing the sequence of coefficients, the bracketed one being the constant term. Thus the lower sequence $(1,-1,1,-2,[2],-1,1)$ beneath the knot 61 shows that its Jones polynomial is $q^{-4}-q^{-3}+q^{-2}-2 q^{-1}+2-q+q^{2}$. The richness of structure of both invariants is immediately clear from the sixteen examples in our table. There are no duplications (except for the Alexander polynomials of the two trefoils, which we put in deliberately to make a point). The arrays of subscripts and superscripts, as well as the roots (which are not shown) and the poles of the Jones invariant (about which almost nothing is known), suggest that the polynomials could encode interesting properties of knots. Notice that $A_{q}(\mathbf{K})$ is symmetric, i.e., $A_{q}(\mathbf{K})=\mathbf{A}_{q^{-1}}(\mathbf{K})$, or, equivalently, the array of coefficients is palendromic. On the other hand, $J_{q}(\mathbf{K})$ is not. Both polynomials take the value 1 at $q=1$. (Remark. The Alexander polynomial is actually 


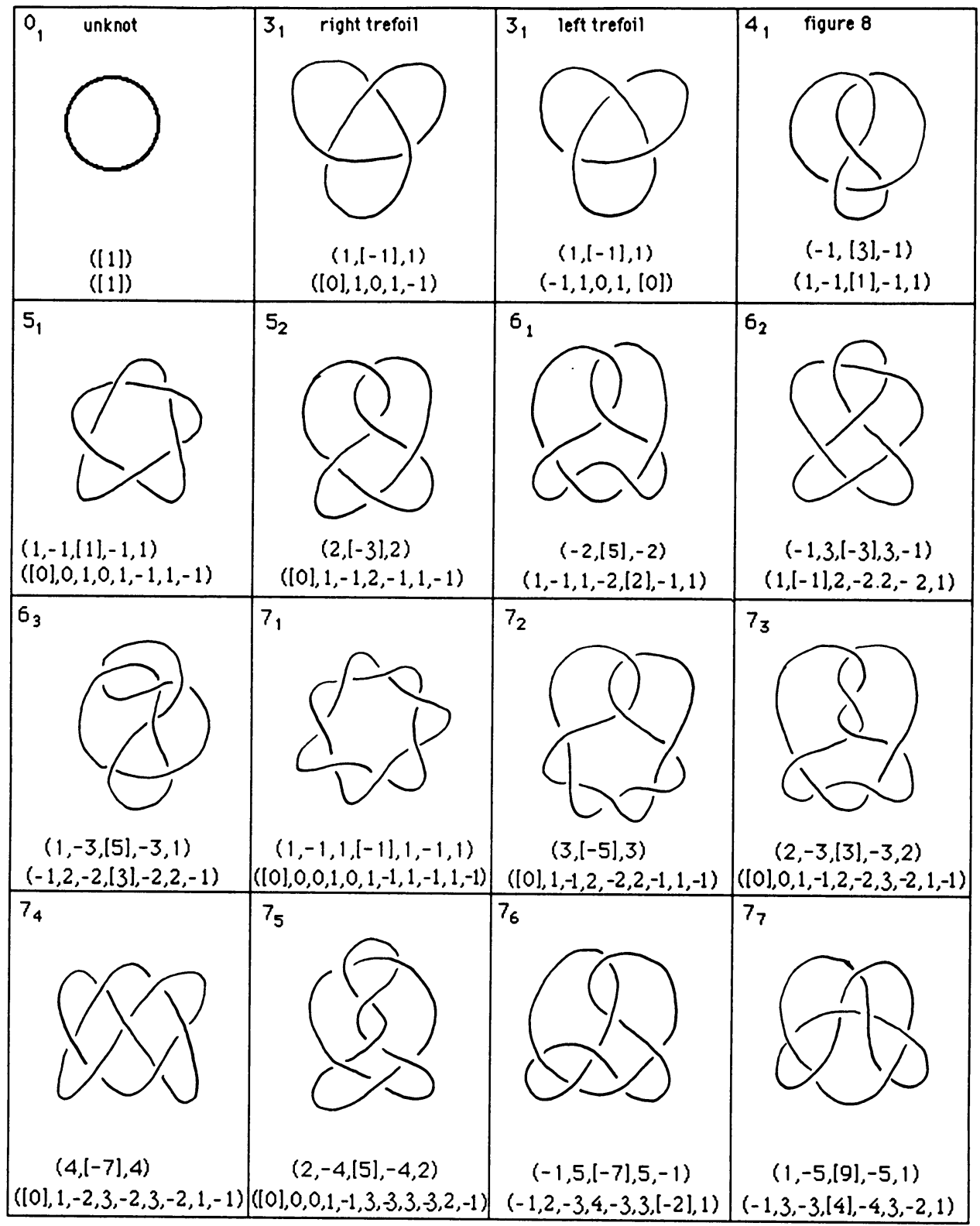

FIGURE 4.

only determined up to \pm multiplicative powers of $q$, and we have chosen to normalize it to stress the symmetry and so that its value at 1 is +1 rather than -1 .)

The knots in our table are all invertible; i.e., there is an isotopy of 3-space which takes the oriented knot to itself with reversed orientation. The first knot in the tables that fails to have that property is $8_{17}$. Neither the Alexander nor the Jones polynomials changes when the orientation on the knot is changed. We shall have more to say about noninvertible knots in $\S 8$.

While we understand the underlying topological meaning of $A_{q}(\mathbf{K})$ very well, 
we are only beginning to understand the topological underpinnings of $J_{q}(\mathbf{K})$. To begin to explain the first assertion, let us go back to one of the earliest problems in knot theory: to what extent does the topological type $\mathbf{X}$ of the complementary space $X=S^{3}-K$ and/or the isomorphism class $\mathbf{G}$ of its fundamental group $G(K)=\pi_{1}\left(X, x_{0}\right)$ suffice to classify knots? The trefoil knot is almost everybody's candidate for the simplest example of a nontrivial knot, so it seems remarkable that, not long after the discovery of the fundamental group of a topological space, Max Dehn [De] succeeded in proving that the trefoil knot and its mirror image had isomorphic groups, but that their knot types were distinct. Dehn's proof is very ingenious! This was the beginning of a long story, with many contributions (e.g., see [Sei, Si, CGL]) which reduced repeatedly the number of distinct knot types which could have homeomorphic complements and/or isomorphic groups, until it was finally proved, very recently, that (i) $\mathbf{X}$ determines $\mathbf{K}$ (see [GL]) and (ii) if $\mathbf{K}$ is prime, then $\mathbf{G}$ determines $\mathbf{K}$ up to unoriented equivalence [Wh]. Thus there are at most four distinct oriented prime knot types which have the same knot group [Wh]. This fact will be important to us shortly.

The knot group $\mathbf{G}$ is finitely presented; however, it is infinite, torsion-free, and (if $\mathbf{K}$ is not the unknot) nonabelian. Its isomorphism class is in general not easily understood via a direct attack on the problem. In such circumstances, the obvious thing to do is to pass to the abelianized group, but unfortunately $G /[G, G] \cong H_{1}(X ; Z)$ is infinite cyclic for all knots, so it is of no use in distinguishing knots. Passing to the covering space $\tilde{X}$ which belongs to $[G, G]$, we note that there is a natural action of the cyclic group $G /[G, G]$ on $\widetilde{X}$ via covering translations. The action makes the homology group $H_{1}(\widetilde{X} ; \mathrm{Z})$ into a $\mathrm{Z}\left[q, q^{-1}\right]$-module, where $q$ is the generator of $G /[G, G]$. This module turns out to be finitely generated. It's the famous Alexander module. While the ring $\mathrm{Z}\left[q, q^{-1}\right]$ is not a PID, relevant aspects of the theory of modules over a PID apply to $H_{1}(\widetilde{X} ; Z)$. In particular, it splits as a direct sum of cyclic modules, the first nontrivial one being $\mathrm{Z}\left[q, q^{-1}\right] / A_{q}(\mathbf{K})$. Thus $A_{q}(\mathbf{K})$ is the generator of the "order ideal", and the smallest nontrivial torsion coefficient in the module $H_{1}(\tilde{X})$. In particular, $A_{q}(\mathbf{K})$ is very clearly an invariant of the knot group.

We regard the above description of $A_{q}(\mathbf{K})$ as an excellent model for what we might wish in a topological invariant of knots. We know precisely what it detects, and so we also know precisely what it fails to detect. For example, it turns out that $\pi_{1}(\widetilde{X})$ is finitely generated if and only if $X$ has the structure of a surface bundle over $S^{1}$, but there is no way to tell definitively from $A_{q}(\mathbf{K})$ whether $\pi_{1}(\tilde{X})$ is or is not finitely generated. On the other hand, if a surface bundle structure exists, the genus of the surface is determined by $A_{q}(\mathbf{K})$. The polynomial $A_{q}(\mathbf{K})$ also generalizes in many ways. For example, there are Alexander invariants of links, also additional Alexander invariants when the Alexander module has more than one torsion coefficient. Moreover, the entire theory generalizes naturally to higher dimensional knots, the generalized invariants playing a central role in that subject.

Returning to the table in Figure 4, we remark that when a knot is replaced by its mirror image (i.e., the orientation on $S^{3}$ is reversed), the Alexander and Jones polynomials $A_{q}(\mathbf{K})$ and $J_{q}(\mathbf{K})$ go over to $A_{q^{-1}}(\mathbf{K})$ and $J_{q^{-1}}(\mathbf{K})$ respectively. As noted earlier, $A_{q}(\mathbf{K})$ is invariant under such a change, but 
from the simplest possible example, the trefoil knot, we see that $J_{q}(\mathbf{K})$ is not. Now recall that $\mathbf{G}$ does not change under changes in the orientation of $S^{3}$. This simple argument shows that $J_{q}(\mathbf{K})$ cannot be a group invariant! Since there are at most four distinct knot types that share the same knot group $\mathbf{G}$, a first wild guess would be that $J_{q}(\mathbf{K})$, which does detect changes in the ambient space orientation (but not in knot orientation), classifies unoriented knot types; but this cannot be true because [Kan] constructs examples of infinitely many distinct prime knot types with the same Jones polynomial. Thus it seems interesting indeed to ask about the underlying topology behind the Jones polynomial. If it is not a knot group invariant, what can it be? We will begin to approach that problem by a circuitous route, taking as a hint the central and very surprising role of "crossing-change formulas" in the subject.

\section{Crossing changes}

A reader who is interested in the history of mathematics will find, on browsing through several of Alexander's Collected Works, that many of his papers end with an intriguing or puzzling comment or remark which, as it turned out with the wisdom of hindsight, hinted at future developments of the subject. For example, in his famous paper on braids [All], which we will discuss in detail in $\S 6$, he proves that every knot or link may be represented as a closed braid. He then remarks (at the end of the paper) that this yields a construction for describing 3-manifolds via their fibered knots; however, he did it long before anyone had considered the concept of a fibered knot! Another example that is of direct interest to us now occurs in [A12], where he reports on the discovery of the Alexander polynomial. In equation (12.2) of that paper we find observations on the relationship between the Alexander polynomials of three links: $K_{p_{+}}, K_{p_{-}}$, and $K_{p_{0}}$, which are defined by diagrams that are identical outside a neighborhood of a particular double point $p$, where they differ in the manner indicated in Figure 5.

The formula which Alexander gives is:

$$
A_{q}\left(\mathbf{K}_{p_{+}}\right)-A_{q}\left(\mathbf{K}_{p_{-}}\right)=(\sqrt{q}-1 / \sqrt{q}) A_{q}\left(\mathbf{K}_{p_{0}}\right) .
$$

This formula passed unnoticed for forty years. (We first learned about Alexander's version of it in 1970 from Mark Kidwell.) Then, in 1968 it was rediscovered, independently, by John Conway [C], who added a new observation: If you require, in addition to (1a), that:

$$
A_{q}(\mathbf{O})=1,
$$

where $\mathbf{O}$ is the unknot, then (1a) and (1b) determine $A_{q}(\mathbf{K})$ on all knots, by a recursive procedure. To see this, the first thing to observe is that if $\mathbf{O}_{\mu}$ is the
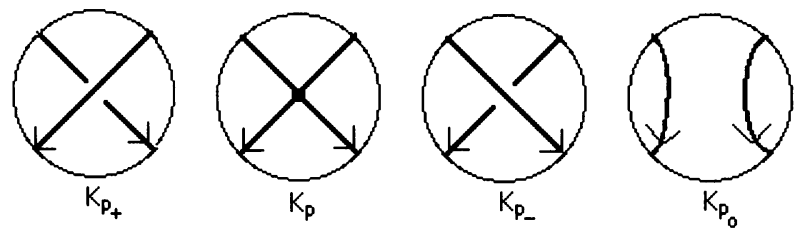

FIGURE 5. Related link diagrams. 

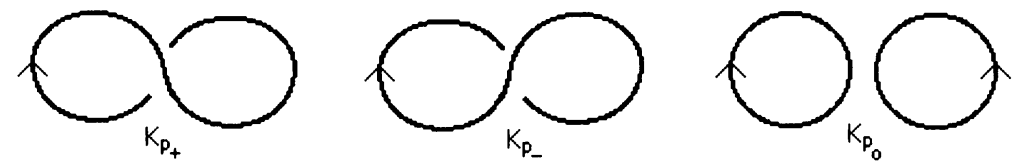

Figure 6. Three related diagrams for the unlink.

$\mu$-component unlink, then we may find related diagrams in which $K_{p_{+}}, K_{p_{-}}$, and $K_{p_{0}}$ represent $\mathbf{O}_{\mu}, \mathbf{O}_{\mu}$, and $\mathbf{O}_{\mu+1}$ respectively, as in Figure 6 .

This fact, in conjunction with (1a), implies that $A_{q}(\mathbf{O})=0$ if $\mu \geq 2$. Next, recall (Figure 1b) that any diagram $K$ for any knot type $\mathbf{K}$ may be changed to a layered diagram which represents the unknot or unlink on the same number of components, by appropriate crossing changes. Induction on the number of crossing changes to the unlink then completes the proof of Conway's result. This led him to far-reaching investigations of the combinatorics of knot diagrams.

While the Jones polynomial was discovered via braid theory (and we shall have more to say about that shortly), Jones noticed, very early in the game, that his polynomial also satisfied a crossing-change formula, vis:

$$
q^{-1} J_{q}\left(\mathbf{K}_{p_{+}}\right)-q J_{q}\left(\mathbf{K}_{p_{-}}\right)=(\sqrt{q}-1 / \sqrt{q}) J_{q}\left(\mathbf{K}_{p_{0}}\right) .
$$

which, via Figure 6 and layered diagrams, may be used, in conjunction with the initial data

$$
J_{q}(\mathbf{O})=1 \text {, }
$$

to compute $J_{q}(\mathbf{K})$ for all knots and links. Motivated by the similarity between (1a)-(1b) and (2a)-(2b), several authors [LM, Ho, PT] were led to consider a more general crossing-change formula, which for our purposes may be described as an infinite sequence of crossing-change formulas:

$$
\begin{aligned}
q^{-n} H_{q, n}\left(\mathbf{K}_{p_{+}}\right)-q^{n} H_{q, n}\left(\mathbf{K}_{p_{-}}\right) & =(\sqrt{q}-1 / \sqrt{q}) H_{q, n}\left(\mathbf{K}_{p_{0}}\right), \\
H_{q, n}(\mathbf{O}) & =1,
\end{aligned}
$$

where $n \in \mathrm{Z}$. It turns out that $(3 \mathrm{a})-(3 \mathrm{~b})$ determine an infinite sequence of one-variable polynomials which in turn extend uniquely to give a two-variable invariant which has since become known as the HOMFLY polynomial [FHL]. Later, (1a)-(3b) were replaced by a more complicated family of crossing-change formulas, yielding the Kauffman polynomial invariant of knots and links [Kau 1]. A unifying principle was discovered (see [Re2]) which yielded still further invariants, for example, the $G_{2}$ invariant of embedded knots, links, and graphs $[\mathrm{Ku}]$. Later, crossing-change formulas were used to determine other polynomial invariants of knotted graphs [Y1] as well.

At this time we know many other polynomial invariants of knots, links, and graphs. In principle, all of them can be defined via generalized crossing-changes, together with initial data. In general, a particular polynomial will be defined by a family of equations which are like (2a) and (3a). It is to be expected that each such equation will relate the invariants of knots which are defined by diagrams which differ in some specified way, in a region which has a fixed number of incoming and outgoing arcs. The ways in which they differ will be more complicated than a simple change in a crossing and "surgery" of the crossing. Thus we have a conundrum: on the one hand, knot and link diagrams 
and crossing-change formulas clearly have much to do with the subject; on the other hand, their role is in many ways puzzling, because we do not seem to be learning as much as we might expect to learn about diagram-related invariants from the polynomials.

We make this last assertion explicit. First, let us define three diagram-related knot invariants:

(i) the minimum crossing number $c(\mathbf{K})$ of a knot,

(ii) the minimum number of crossing changes $u(\mathbf{K})$, i.e., to the unknot, and

(iii) the minimum number of Seifert circles $s(\mathbf{K})$,

where each of these invariants is to be minimized over all possible knot diagrams. (Aside. For the reader who is unfamiliar with the concept of Seifert circles, we note that by [Y2] the integer $s(\mathbf{K})$ may also be defined to be the braid index of a knot or link, i.e., the smallest integer $s$ such that $\mathbf{K}$ may be represented as a closed $s$-braid. If the reader is also not familiar with closed braids, he or she might wish to peek ahead to $\S 3$.) All of them satisfy inequalities which are detected by knot polynomials. For example, the Morton-Franks-Williams inequality places upper and lower bounds on $s(\mathbf{K})$ [Mo2, FW]. Also, the Bennequin inequality, recently proved by Menasco [Me], gives a lower bound for $u(\mathbf{K})$ which can be detected by the one-variable Jones polynomial. As another example, the one-variable Jones polynomial was a major tool in the proof of the Tait conjecture (see [Kau2, Mu]), which relates to the definitive determination of $c(\mathbf{K})$ for the special case of alternating knots which are defined by alternating diagrams. On the other hand, there are examples which show that none of the inequalities mentioned above can be equalities for all knots. Indeed, at this writing we do not have a definitive method for computing any of these very intuitive diagram-related knot and link-type invariants.

\section{R-MATRIX REPRESENTATIONS OF THE BRAID GROUP}

One of the very striking successes of the past eight years is that, after a period during which new polynomial invariants of knots and links were being discovered at an alarming rate (e.g., see [WAD]), order came out of chaos and a unifying principle emerged which gave a fairly complete description of the new invariants; we study it in this section. We remark that this description may be given in at least two mutually equivalent ways: the first is via the algebras of [Jo4, BW, Kal] and the cabling construction of [Mu, We2]; the second is via the theory of R-matrices, as we shall do here.

Our story begins with the by-now familiar notion of an $n$-braid [Art]. See Figure 7 (a) for a picture, when $n=3$. Our $n$-braid is to be regarded as living in a slab of 3-space $\mathbb{R}^{2} \times I \subset \mathbb{R}^{3}$. It consists of $n$ interwoven oriented strings which join $n$ points, labeled $1,2, \ldots, n$, in the plane $\mathbb{R}^{2} \times\{0\}$ with corresponding points in $\mathbb{R}^{2} \times\{1\}$, intersecting each intermediate plane $\mathbb{R}^{2} \times\{t\}$ in exactly $n$ points. Two braids are equivalent if there is an isotopy of one to the other which preserves the initial and end point of each string, fixes each plane $\mathbb{R}^{2} \times\{t\}$ setwise, and never allows two strings to intersect. Multiplication is by juxtaposition, erasure of the middle plane, and rescaling. Closed braids are obtained from (open) braids by joining the initial point of each strand, in $\mathbb{R}^{3}$, to the corresponding end point, in the manner illustrated in Figure $7(\mathrm{~b})$, so that if one thinks of the closed braid as wrapping around the $z$-axis, it meets each plane $\theta=$ constant in exactly $n$ points. A famous theorem of Alexander [Al1] 

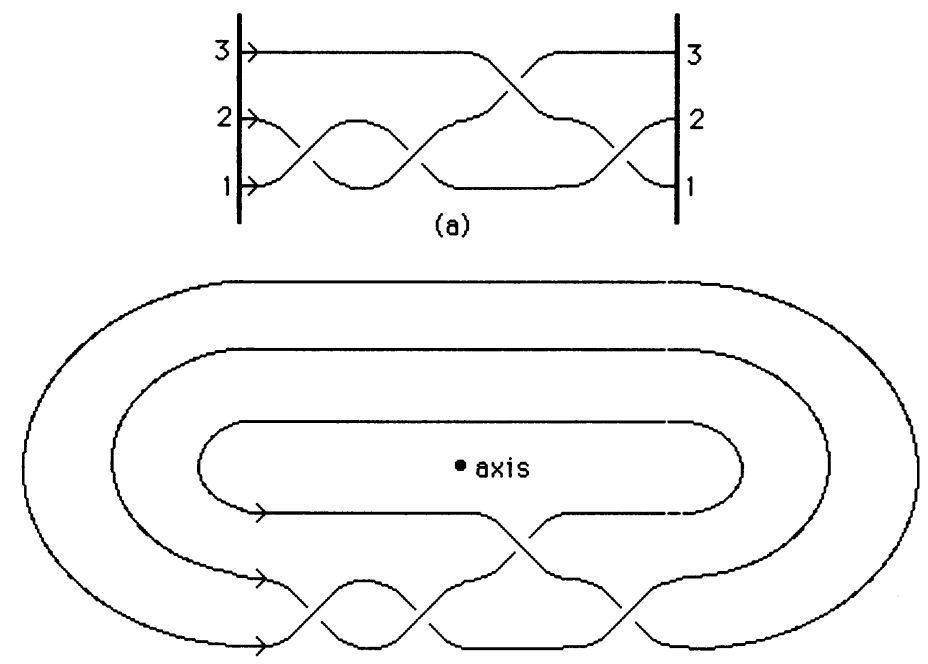

(b)

FIGURE 7. Braids.

asserts that every knot or link may be so represented, for some $n$. (Remark. Lemma 1 of $\S 6$ sketches a generalization of Alexander's original proof.) In fact, if $K$ is our oriented link, we may choose any oriented $\mathbb{R}^{1} \subset \mathbb{R}^{3}$ which is disjoint from $K$ and modify $K$ by isotopy so that this copy of $\mathbb{R}^{1}$ is the braid axis.

The braid group $B_{n}$ is generated by the elementary braids $\sigma_{1}, \ldots, \sigma_{n-1}$ illustrated in Figure 8. For example, the braid in Figure 7(a) may be described, using the generators given in Figure 8, by the word $\sigma_{1}^{-2} \sigma_{2} \sigma_{1}^{-1}$. Defining relations in $B_{n}$ are:

$$
\begin{gathered}
\sigma_{i} \sigma_{j}=\sigma_{j} \sigma_{i} \quad \text { if }|i-j| \geq 2, \\
\sigma_{i} \sigma_{j} \sigma_{i}=\sigma_{j} \sigma_{i} \sigma_{j} \text { if }|i-j|=1,
\end{gathered}
$$

We will refer to these as the braid relations.

Let $B_{\infty}$ be the disjoint union of the braid groups $B_{1}, B_{2}, \ldots$ Define $\beta$, $\beta^{*} \in B_{\infty}$ to be Markov-equivalent if the closed braids $\hat{\beta}, \hat{\beta}^{*}$ which they define represent the same link type. Markov's theorem, announced in [Mar]
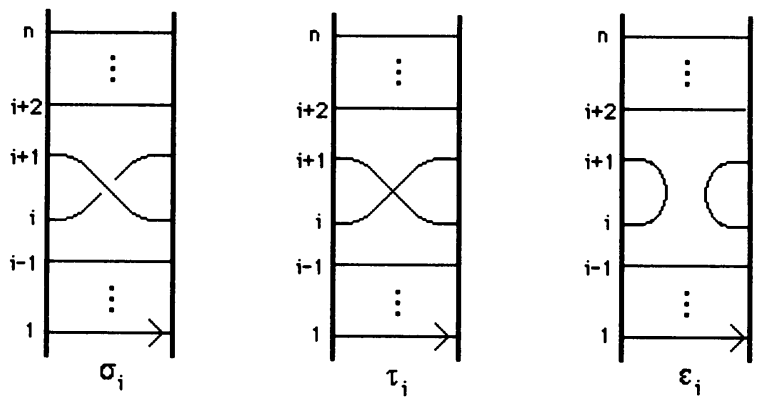

FIGURE 8. Elementary braids, singular braids, and tangles. 
and proved forty years later in [Bi], asserts that Markov equivalence is generated by conjugacy in each $B_{n}$ and the map $B_{n} \rightarrow B_{n+1}$ which takes a word $W\left(\sigma_{1}, \ldots \sigma_{n-1}\right)$ to $W\left(\sigma_{1}, \ldots \sigma_{n-1}\right) \sigma_{n}^{ \pm 1}$. We call the latter Markov's second move. The examples in Figures $7(\mathrm{a}), 7(\mathrm{~b})$ may be used to illustrate Markov equivalence. The 3-braid $\sigma_{1}^{-2} \sigma_{2} \sigma_{1}^{-1}$ shown there is conjugate in $B_{3}$ to $\sigma_{1}^{-3} \sigma_{2}$, which may be modified to the 2-braid $\sigma_{1}^{-3}$ using Markov's second move, so that $\sigma_{1}^{-3} \in B_{2}$ is Markov equivalent to $\sigma_{1}^{-2} \sigma_{2} \sigma_{1}^{-1} \in B_{3}$.

Let $\left\{\rho_{n}: B_{n} \rightarrow \mathrm{GL}_{m_{n}}(\mathscr{E}) ; n=1,2,3, \ldots\right\}$ be a family of matrix representations of $B_{n}$ over a ring $\mathscr{E}$ with 1 . Let $i_{n}: B_{n} \rightarrow B_{n+1}$ be the natural inclusion map which takes $B_{n}$ to the subgroup of $B_{n+1}$ of braids on the first $n$ strings. A linear function $\operatorname{tr}: \rho_{n}\left(B_{n}\right) \rightarrow \mathscr{E}$ is said to be a Markov trace if:

(i) $\operatorname{tr}(1)=1$,

(ii) $\operatorname{tr}\left(\rho_{n}(\alpha \beta)\right)=\operatorname{tr}\left(\rho_{n}(\beta \alpha)\right) \quad \forall \alpha, \beta \in B_{n}$,

(iii) $\exists z \in \mathscr{E}$ such that if $\beta \in i_{n}\left(B_{n}\right)$, then $\operatorname{tr}\left(\rho_{n+1}\left(\beta \sigma_{n}^{ \pm 1}\right)\right)=(z)\left(\operatorname{tr}\left(\rho_{n}(\beta)\right)\right.$.

In particular, by setting $\beta=1$ in (iii) we see that $z=\operatorname{tr}\left(\rho_{n}\left(\sigma_{n-1}^{ \pm 1}\right)\right)$. In view of Markov's theorem, it is immediate that every family of representations of $B_{n}$ which supports a Markov trace determines a link type invariant $F\left(\mathbf{K}_{\beta}\right)$ of the link type $\mathbf{K}_{\beta}$ of the closed braid $\hat{\beta}$, defined by

$$
F\left(\mathbf{K}_{\beta}\right)=z^{1-n} \operatorname{tr}\left(\rho_{n}(\beta)\right) .
$$

Later, we will need to ask what happens to $(5)$ if we rescale the representation, so we set the stage for the modifications now. Suppose that $\rho=\rho_{n}$ is a representation of $B_{n}$ in $\mathrm{GL}_{m}(\mathscr{E})$ which supports a function $\operatorname{tr}$ which satisfies (i)-(iii). Let $\gamma$ be any invertible element in $\mathscr{E}$. Then we may define a new representation $\rho^{\prime}$ by the rule $\rho^{\prime}\left(\sigma_{i}\right)=\gamma \rho\left(\sigma_{i}\right)$ for each $i=1, \ldots, n$. It is easy to see that $\rho^{\prime}$ is a representation if and only if $\rho$ is, because (4a) and (4b) are satisfied for one if and only if they are satisfied for the other. Properties (i) and (ii) will continue to hold if we replace $\rho$ by $\rho^{\prime}$, but (iii) will be modified because the traces of $\sigma_{i}$ and $\sigma_{i}^{-1}$ will not be the same in the new representation. To determine the effect of the change on equation (5), let $z^{\prime}=\operatorname{tr}\left(\rho^{\prime}\left(\sigma_{i}\right)\right)=\gamma \operatorname{tr}\left(\rho\left(\sigma_{i}\right)\right)=\gamma z$, so $z=\gamma^{-1} z^{\prime}$. Choose any $\beta \in B_{n}$. Then $\beta$ may be expressed as a word $\sigma_{\mu_{1}}^{\varepsilon_{1}} \sigma_{\mu_{2}}^{\varepsilon_{2}} \cdots \sigma_{\mu_{r}}^{\varepsilon_{r}}$ in the generators, where each $\varepsilon_{j}= \pm 1$. Let $\varepsilon(\beta)=\sum_{i}^{r} \varepsilon_{i}$. Then equation (5) will be replaced by

$$
F\left(\mathbf{K}_{\beta}\right)=\gamma^{-\varepsilon(\beta)}\left(z^{\prime}\right)^{1-n} \operatorname{tr}\left(\rho_{n}^{\prime}(\beta)\right) .
$$

Thus the existence of a link type invariant will not be changed if we rescale the representation by introducing the invertible element $\gamma$. Similar considerations apply if we rescale the trace function. For example, instead of requiring that $\operatorname{tr}(1)=1$, we could require that if $1_{n}$ denotes the identity element in $B_{n}$, then $\operatorname{tr}\left(1_{n}\right)=z^{n-1}$, in which case the factor $z^{1-n}$ in equation (5) would vanish.

The invariant which is defined by equation $(5)$ or $\left(5^{\prime}\right)$ depends directly on the traces of matrices in a finite-dimensional matrix representation $\rho_{n}$ of the braid group $B_{n}$. Any such representation is determined by its values on the generators $\sigma_{i}$, and since these are finite-dimensional matrices they satisfy their characteristic polynomials, yielding polynomial identities. From the point of view of this section, such identities are one source of the crossing-change formulas we mentioned earlier, in $\S 2$. Another source will be trace identities, which 
always exist in matrix groups. (See, e.g., [Pr].) In general one needs many such identities (i.e., polynomial equations satisfied by the images of various special braid words) to obtain axioms which suffice to determine a link type invariant.

In the summer of 1984 , almost eight years ago to the day from this writing, this author met with Vaughan Jones to discuss possible ramifications of a discovery Jones had made in [Jo2] of a new family of matrix representations of $B_{n}$, in conjunction with his earlier studies of type $\mathrm{II}_{1}$ factors and their subfactors in von Neumann algebras. Before that time, there was essentially only one matrix representation of the braid group which had a chance of being faithful and had been studied in any detail — the Burau representation (see [Bi]). The knot invariant which was associated to that representation was the Alexander polynomial. Jones had shown that his representations contained the Burau representation as a proper summand. Thus his representations were new and interesting. Also, they supported an interesting trace function. As it developed [Jo3], his trace functions were Markov traces. If $\rho_{n}$ is taken to be the Jones's representation of $B_{n}$, the invariant we called $F\left(\mathbf{K}_{\beta}\right)$ in (5) is the one-variable Jones polynomial $J_{q}(\mathbf{K})$.

Wandering along a bypath. The reader who is pressed for time may wish to omit this detour. We interrupt our main argument to discuss how the Jones representations were discovered, because it is very interesting to see how an almost chance discovery of an unexpected relationship between two widely separated areas of mathematics had ramifications which promise to keep mathematicians busy at work for years to come! Let $M$ denote a von Neumann algebra. Thus $M$ is an algebra of bounded operators acting on a Hilbert space $\mathscr{H}$. The algebra $M$ is called a factor if its center consists only of scalar multiples of the identity. The factor is type $\mathrm{II}_{1}$ if it admits a linear functional $\operatorname{tr}: M \rightarrow \mathbb{C}$, which satisfies:

(i) $\operatorname{tr}(1)=1$,

(ii) $\operatorname{tr}(x y)=\operatorname{tr}(y x) \quad \forall x, y \in M$,

and a positivity condition which shall not concern us here. It is known that the trace is unique, in the sense that it is the only linear form satisfying (i) and (ii). An old discovery of Murray and von Neumann was that factors of type $\mathrm{II}_{1}$ provide a type of "scale" by which one can measure the dimension $\operatorname{dim}_{M} \mathscr{H}$ of the Hilbert space $\mathscr{H}$. The notion of dimension which occurs here generalizes the familiar notion of integer-valued dimensions, because for appropriate $M$ and $\mathscr{H}$ it can be any nonnegative real number or infinity. The starting point of Jones's work was the following question: if $M_{1}$ is a type $\mathrm{II}_{1}$ factor and if $M_{0} \subset M_{1}$ is a subfactor, is there any restriction on the real numbers $\lambda$ which occur as the ratio $\lambda=\operatorname{dim}_{M_{0}} \mathscr{H} / \operatorname{dim}_{M_{1}} \mathscr{H}$ ?

The question has the flavor of questions one studies in Galois theory. On the face of it, there was no reason to think that $\lambda$ could not take on any value in $[1, \infty]$, so Jones's answer came as a complete surprise. He called $\lambda$ the index [ $M_{1}: M_{0}$ ] of $M_{0}$ in $M_{1}$ and proved a type of rigidity theorem about it:

The Jones Index Theorem. If $M_{1}$ is a $\mathrm{II}_{1}$ factor and $M_{0}$ a subfactor, then the possible values of the index $\lambda$ are restricted to $[4, \infty] \cup\left[4 \cos ^{2}(\pi / p)\right]$, where $p \geq 3$ is a natural number. Moreover, each such real number occurs for some pair $M_{0}, M_{1}$. 
We now sketch the idea of Jones's proof, which is to be found in [Jo1]. Jones begins with the type $\mathrm{II}_{1}$ factor $M_{1}$ and a subfactor $M_{0}$. There is also a tiny bit of additional structure: In this setting there exists a map $e_{1}: M_{1} \rightarrow M_{0}$, known as the conditional expectation of $M_{1}$ on $M_{0}$. The map $e_{1}$ is a projection, i.e., $e_{1}^{2}=e_{1}$. His first step is to prove that the ratio $\lambda$ is independent of the choice of the Hilbert space $\mathscr{H}$. This allows him to choose an appropriate $\mathscr{H}$ so that the algebra $M_{2}=\left\langle M_{1}, e_{1}\right\rangle$ generated by $M_{1}$ and $e_{1}$ makes sense. He then investigates $M_{2}$ and proves that it is another type II $_{1}$ factor, which contains $M_{1}$ as a subfactor; moreover, $\left|M_{2}: M_{1}\right|=\left|M_{1}: M_{0}\right|=\lambda$. Having in hand another type II $_{1}$ factor, i.e., $M_{2}$ and its subfactor $M_{1}$, there is also a trace on $M_{2}$ which (by the uniqueness of the trace) coincides with the trace on $M_{1}$ when it is restricted to $M_{1}$ and another conditional expectation $e_{2}: M_{2} \rightarrow M_{1}$. This allows Jones to iterate the construction and to build algebras $M_{1}, M_{2}, \ldots$ and from them a family of algebras:

$$
\mathscr{A}_{n}=\left\langle 1, e_{1}, \ldots, e_{n-1}\right\rangle \subset M_{n}, \quad n=1,2,3, \ldots
$$

We now replace the $e_{k}$ 's by a new set of generators which are units, defining $g_{k}=q e_{k}-1+e_{k}$, where $(1-q)\left(1-q^{-1}\right)=1 / \lambda$. The $g_{k}$ 's generate $\mathscr{A}_{n}$, because the $e_{k}$ 's do, and we can solve for the $e_{k}$ 's in terms of the $g_{k}$ 's. Thus

$$
\mathscr{A}_{n}=\mathscr{A}_{n}(q)=\left\langle 1, g_{1}, \ldots, g_{n-1}\right\rangle,
$$

and we have a tower of algebras, ordered by inclusion:

$$
\mathscr{A}_{1}(q) \subset \mathscr{A}_{2}(q) \subset \mathscr{A}_{3}(q) \subset \cdots .
$$

The parameter $q$, which replaces the index $\lambda$, is the quantity now under investigation. The $g_{i}$ 's turn out to be invertible and to satisfy the braid relations (4a)-(4b), so that there is a homomorphism from $B_{n}$ to $\mathscr{A}_{n}$, defined by mapping the elementary braid $\sigma_{i}$ to $g_{i}$. The parameter $q$ is woven into the construction of the tower. Defining relations in $\mathscr{A}_{n}(q)$ depend upon $q$, for example, the relation $g_{i}^{2}=(q-1) g_{i}+q$ holds. Recall that, since $M_{n}$ is type $\mathrm{II}_{1}$, it supports a unique trace, and, since $\mathscr{A}_{n}$ is a subalgebra, it does too, by restriction. This trace is a Markov trace! Jones's proof of the Index Theorem is concluded when he shows that the infinite sequence of algebras $\mathscr{A}_{n}(q)$, with the given trace, could not exist if $q$ did not satisfy the stated restrictions.

Thus the "independent variable" in the Jones polynomial is essentially the index of a type II $_{1}$ subfactor in a type II $_{1}$ factor! Its discovery opened a new chapter in knot and link theory.

Back to the main road. We now describe a method, discovered by Jones (see the discussion of vertex models in [Jo5]) but first worked out in full detail by Turaev in [Tu], which can be applied to give, in a unified setting, every generalized Jones invariant via a Markov trace on an appropriate matrix representation of $B_{n}$. As before, $\mathscr{E}$ is a ring with 1 . Let $V$ be a free $\mathscr{E}$-module of rank $m \geq 1$. For each $n \geq 1$ let $V^{\otimes n}$ denote the $n$-fold tensor product $V \otimes_{\mathscr{E}} \cdots \otimes_{\mathscr{E}} V$. Choose a basis $v_{1}, \ldots, v_{m}$ for $V$, and choose a corresponding basis $\left\{v_{i_{1}} \otimes \cdots \otimes v_{i_{n}} ; 1 \leq\right.$ $\left.i_{1}, \ldots, i_{n} \leq m\right\}$ for $V^{\otimes n}$. An $\mathscr{E}$-linear isomorphism $f$ of $V^{\otimes n}$ may then be represented by an $m^{n}$-dimensional matrix $\left(f_{i_{1} \cdots i_{n}}^{j_{1} \cdots j_{n}}\right)$ over $\mathscr{E}$, where the $i_{k}$ 's (resp. $j_{k}$ 's) are row (resp. column) indices. 
The family of representations of $B_{n}$ which we now describe have a very special form. They are completely determined by an $\mathscr{E}$-linear isomorphism $\mathrm{R}: V^{\otimes 2} \rightarrow V^{\otimes 2}$ with matrix $\left[\mathrm{R}_{i_{1} i_{2}}^{j_{1} j_{1}}\right]$ as above. Let $I_{V}$ denote the identity map on the vector space $V$. The representation $\rho_{n, \mathrm{R}}: B_{n} \rightarrow \mathrm{GL}_{m^{n}}(\mathscr{E})$ that we need is defined by

$$
\rho_{n, \mathrm{R}}\left(\sigma_{i}\right)=I_{V} \otimes \cdots \otimes I_{V} \otimes \mathrm{R} \otimes I_{V} \otimes \cdots \otimes I_{V}
$$

where $\mathrm{R}$ acts on the $i$ th and $(i+1)$ st copies of $V$ in $V^{\otimes n}$. Thus, if we know how $\mathrm{R}$ acts on $V^{\otimes 2}$, we know $\rho_{n, \mathrm{R}}$ for every natural number $n$.

What properties must $\mathrm{R}$ satisfy for $\rho_{n, \mathrm{R}}$ to be a representation? The first thing to notice is that, if $|i-j| \geq 2$, the nontrivial parts of $\rho_{n, \mathrm{R}}\left(\sigma_{i}\right)$ and $\rho_{n, \mathrm{R}}\left(\sigma_{j}\right)$ will not interfere with one another, so (4a) is satisfied by construction, independently of the choice of $R$. As for (4b), it is clear that we only need to look at the actions of $\mathrm{R} \otimes I_{V}$ and $I_{V} \otimes \mathrm{R}$ on $V^{\otimes 3}$, for if

$$
\left(\mathrm{R} \otimes I_{V}\right)\left(I_{V} \otimes \mathrm{R}\right)\left(\mathrm{R} \otimes I_{V}\right)=\left(I_{V} \otimes \mathrm{R}\right)\left(\mathrm{R} \otimes I_{V}\right)\left(I_{V} \otimes \mathrm{R}\right),
$$

then (4b) will be satisfied for every pair $\sigma_{i}, \sigma_{j}$ with $|i-j|=1$. Thus equation (7) is the clue to the construction. It is known as the quantum Yang-Baxter equation ( $\mathrm{QYBE})$. Note that if $\mathrm{R}$ satisfies the $\mathrm{QYBE}$ equation, then $\alpha \mathrm{R}$ will too, for every invertible element $\alpha \in \mathscr{E}$. By our earlier observations (see the discussion of equations $(5)$ and $\left(5^{\prime}\right)$ ), if the representation determined by $R$ can be used to define a link invariant, then the same will be true if $R$ is replaced by $\alpha \mathrm{R}$. We will use this fact shortly, modifying our choice of $\mathrm{R}$ by composing with an invertible scalar, whenever it simplifies the equations to do so.

Caution. One must distinguish between (7), which we call the QYBE, and a closely related equation $\left(7^{*}\right)$, which is also referred to as the QYBE. To explain the latter and to show that both forms have the same geometric meaning, let us return momentarily to braids. Our elementary braid generators could have just as well been called $\sigma_{i, i+1}$ to stress the fact that the nontrivial part of the braid involves the adjacent strands $i$ and $i+1$. Relation (4b) in $B_{3}$ would then be

$$
\sigma_{12} \sigma_{23} \sigma_{12}=\sigma_{23} \sigma_{12} \sigma_{12} \text {. }
$$

An alternative way to number the braid generators would be to color the strands with three colors, labeled $1,2,3$ and to let $\check{\sigma}_{i, j}$ denote a positive crossing between the strands of color $i$ and color $j$, when those two colors are adjacent in the braid projection. With that convention, we see from the bottom left picture in Figure 14 on page 277 (which is the braid version of Reidemeister's move III) that ( $4 b)$ would be

$$
\check{\sigma}_{12} \check{\sigma}_{13} \check{\sigma}_{23}=\check{\sigma}_{23} \check{\sigma}_{13} \check{\sigma}_{12} \text {. }
$$

Going back to operators acting on $V^{\otimes 3}$, let $\mathrm{R}_{12}=\mathrm{R} \otimes I_{V}$ and let $\mathrm{R}_{23}=I_{V} \otimes \mathrm{R}$. Then equation (7) may be rewritten as

$$
\mathbf{R}_{12} \mathbf{R}_{23} \mathbf{R}_{12}=\mathbf{R}_{23} \mathbf{R}_{12} \mathbf{R}_{23} \text {. }
$$

Now let $\check{\mathbf{R}}_{i j}$ be the image of $\mathbf{R}_{i j}$ under the automorphism of $V^{\otimes 3}$ induced by the permutation of $\{1,2,3\}$ which maps 1 to $i$ and 2 to $j$. The alternative form of the QYBE is

$$
\check{\mathbf{R}}_{12} \check{\mathbf{R}}_{13} \check{\mathbf{R}}_{23}=\check{\mathbf{R}}_{23} \check{\mathbf{R}}_{13} \check{\mathbf{R}}_{12} \text {. }
$$


In this form, it occurs in nature in many ways, for example, in the theory of exactly solvable models in statistical mechanics, where it appears as the startriangle relation [Bax].

Not long after the discovery that the Jones polynomial generalized to the HOMFLY and Kauffman polynomials, workers in the area began to discover other, isolated cases of generalizations, all relating to existing known solutions to $\left(7^{*}\right)$. Then a coherent theory emerged. It turns out that solutions to $\left(7^{*}\right)$, and hence (7), can be constructed with the help of known solutions to yet a third equation-the classical Yang-Baxter equation (CYBE). To explain that equation, let $\mathscr{L}$ be a Lie algebra and let $\mathrm{r} \in \mathscr{L}^{\otimes 2}$. Let $\mathrm{r}_{12}$ be $\mathrm{r} \otimes 1 \in \mathscr{L}^{\otimes 3}$ and let $\mathrm{r}_{i j}$ be the image of $\mathrm{r}_{12}$ under the automorphism of $\mathscr{L} \otimes 3$ induced by the permutation of $\{1,2,3\}$ which sends 1 to $i$ and 2 to $j$. Then $\mathrm{r}$ is said to be a solution to the CYBE if the following holds:

$$
\left[r_{12}, r_{23}\right]+\left[r_{13}, r_{23}\right]+\left[r_{12}, r_{13}\right]=0
$$

The theory of the CYBE is well understood, its solutions having been essentially classified. Two good references on the subject, both with extensive bibliographies, are [Sem] and [BD]. The possibility of "quantization", i.e., passage from solutions to the CYBE $\left(7^{* *}\right)$ to those for the QYBE $\left(7^{*}\right)$ was proved by Drinfeld in a series of papers, starting with [Dr1]. See [Dr2] for a review of the subject and another very extensive bibliography, including the definition of a quantum group and a development of the relevance of the theory of quantum groups to this matter. That theory has much to do with the representations of simple Lie algebras. Explicit solutions to $\left(7^{*}\right)$ which are associated to the fundamental representations of the nonexceptional classical Lie algebras may be found in the work of Jimbo ([Ji1] and especially [Ji2]). See [WX] for a discussion of the problem of finding "classical" solutions to the "quantum" equation $\left(7^{*}\right)$.

Now, representations of $B_{n}$ do not always support a Markov trace. To obtain a Markov trace from the representation defined in (6), where $R$ satisfies (7), one needs additional data in the form of an enhancement of $R[\operatorname{Re} 1]$. See Theorem 2.3.1 of [Tu]. The enhancement is a choice of invertible elements $\mu_{1}, \ldots, \mu_{m} \in \mathscr{E}$ which determine a matrix $\mu=\operatorname{diag}\left(\mu_{1}, \ldots, \mu_{m}\right)$ such that

$$
\begin{gathered}
\mu \otimes \mu \text { commutes with } \mathbf{R}=\left[\mathbf{R}_{i_{1} i_{2}}^{j_{1} j_{2}}\right], \\
\sum_{j=1}^{m}\left(\mathbf{R}^{ \pm 1}\right)_{i, j}^{k, j} \cdot \mu_{j}=\delta_{i, k}(\text { Kroneker symbol }) .
\end{gathered}
$$

( $A$ remark for the experts. Theorem 2.3.1 of [Tu] contains two factors, $\alpha$ and $\beta$. We have chosen them to be 1 . This possibility is discussed in Remark (i), $\S 3.3$ of [Tu]. We may do this because, as noted earlier, if $R$ is a solution to the QYBE, then $\alpha \mathrm{R}$ is too, for any invertible $\alpha \in \mathscr{E}$.)

As it turns out, there are invertible elements $\mu_{1}, \ldots, \mu_{m} \in \mathscr{E}$ associated naturally to every solution to the QYBE which comes from quantum groups and has the property that $(8 \mathrm{a})$ is true. This is proved in [Ros, $\operatorname{Re} 2, \mathrm{RT} 1]$. It is also known that if the $R$-matrix comes from an irreducible representation of a quantum group, then (8b) is true with $\delta_{i, k}$ replaced by $\alpha^{-1} \delta_{i, k}$ for some invertible element $\alpha \in \mathscr{E}$. Notice that (7) and (8a) still hold if $\mathrm{R}$ is replaced 
by $\alpha \mathrm{R}$. Thus, possibly replacing $\mathrm{R}$ by $\alpha \mathrm{R}$, every solution to the QYBE coming from quantum groups admits an enhancement.

Finally, we describe how generalized Jones invariants are constructed out of the data $\left(\mathrm{R}, n, \mu_{1}, \ldots, \mu_{m}\right)$. Let $\rho=\rho_{n, \mathrm{R}}$. Let $\mu^{\otimes n}$ denote the mapping $\mu \otimes \cdots \otimes \mu: V^{\otimes n} \rightarrow V^{\otimes n}$. If $\beta \in B_{n}$, define $\operatorname{tr}(\rho(\beta))$ to be the ordinary matrix trace of $\rho(\beta) \cdot \mu^{\otimes n}$. Notice that this implies that, if $1_{n}$ denotes the identity element in $B_{n}$, then $\operatorname{tr}\left(1_{n}\right)=\left(\mu_{1}+\cdots+\mu_{m}\right)^{n}$ is the matrix trace of $\mu^{\otimes n}$. With this rescaling of the trace the factor $z$ in equation (5) goes away (see the discussion following the statement of equation (5)). The link invariant $F\left(\mathbf{K}_{\beta}\right)$ is given explicitly as

$$
F\left(\mathbf{K}_{\beta}\right)=\operatorname{tr}\left(\rho(\beta) \cdot \mu^{\otimes n}\right) .
$$

We leave it to the reader to verify that $(8 \mathrm{a})$ and $(8 \mathrm{~b})$ ensure that $F\left(\mathbf{K}_{\beta}\right)$ in (9) is invariant under Markov's two moves. (Alternatively, see Theorem 3.12 of [Tu] for a proof.) The ring $\mathscr{E}$ (to the best of our knowledge) will always be a ring of Laurent polynomials over the integers, the variable being some root of $q$. In the special case of the HOMFLY polynomial (as defined in (3a) and (3b)), the function $F\left(\mathbf{K}_{\beta}\right)$ of (9) turns out to depend only on $q^{ \pm 1}$ when $\mathbf{K}_{\beta}$ is a knot. For links, square roots generally occur. The invariant $F\left(\mathbf{K}_{\beta}\right)$ in (9) is normalized so that $F(\mathbf{O})=\mu_{1}+\cdots+\mu_{m}$.

The HOMFLY and Kauffman polynomials, which are central to the subject, are related to the fundamental representations of the nonexceptional Lie algebras of type $A_{n}^{1}$ and $B_{n}^{1}, C_{n}^{1}, D_{n}^{1}, A_{n}^{2}$. Other knot and link polynomials, for example, the ones in [WAD], turn out to be the HOMFLY and Kauffman polynomials of various cables on simple (noncabled) knots and links. Yet other completely new polynomials arise out of the representations of the remaining nonexceptional Lie algebras and the exceptional Lie algebras. For example, see $[\mathrm{Ku}]$ for the case of the exceptional Lie algebra $G_{2}$ from the point of view of crossing-change formulas and [Kal] for an interpretation of Kuperberg's polynomial via traces on representations of $B_{n}$. We understand that the representations corresponding to the algebras $E_{6}, E_{7}, E_{8}$ have also been constructed, but we do not have a reference. The important point is that it is possible to do so.

Summarizing our results in this section, we have described a very general construction which leads to the construction of vast families of knot and link invariants. The proof, via Markov's theorem, that they do not depend on the choice of the representative braid $\beta \in B_{\infty}$ is straightforward and convincing. Unfortunately, however, neither the construction (which depends upon the combinatorial properties needed to satisfy $(7)$ or $\left(7^{*}\right)$ and $\left.(8)\right)$ nor the proofs we know of Markov's theorem ([Be, $\mathrm{Bi}, \mathrm{Mol}]$ and most recently [Mak]) give the slightest hint as to the underlying topology; moreover, the constructions which actually yield all possible solutions to (7) and (8) do not change that situation at all. The crossing-change formulas in $\S 2$ give even less insight. Thus we can compute the simplest of the invariants by hand and quickly fill pages with the coefficients and exponents of $J_{q}(\mathbf{K})$ for not-too-complicated knots without having the slightest idea what they mean.

\section{A SPACE OF ALl KNOTS}

To proceed further, we need to change our point of view. The Alexander polynomial, as we have seen, contains information about the topology of the 
complement of a single knot. We now describe yet another method of constructing knot invariants, discovered in 1989, which yields information about the topology of an appropriately defined space of all knots.

Following methods pioneered by Arnold (e.g., see the introduction to [Arn1]) and developed for the case of knots in 3-space by Vassiliev [V], we begin by introducing a change which is very natural in mathematics, shifting our attention from the knot $K$, which is the image of $S^{1}$ under an embedding $\phi: S^{1} \rightarrow S^{3}$, to the embedding $\phi$ itself. A knot type $\mathbf{K}$ thus becomes an equivalence class $\{\phi\}$ of embeddings of $S^{1}$ into $S^{3}$. The space of all such equivalence classes of embeddings is disconnected, with a component for each smooth knot type, and the next step is to enlarge it to a connected space. With that is mind, we pass from embeddings to smooth maps, thereby admitting maps which have various types of singularities. Let $\widetilde{\mathscr{M}}$ be the space of all smooth maps from $S^{1}$ to $S^{3}$. This space is connected and contains all knot types. Our space will remain connected and will contain all knot types if we place two mild restrictions on our maps. Let $\mathscr{M}$ denote the collection of all $\phi \in \widetilde{\mathscr{M}}$ such that $\phi\left(S^{1}\right)$ passes through a fixed point $*$ and is tangent to a fixed direction at $*$. The space $\mathscr{M}$ has some pleasant properties, the main one being that it can be approximated by certain affine spaces, and these affine spaces contain representatives of all knot types. The walls between distinct chambers in $\mathscr{M}$ constitute the discriminant $\Sigma$, i.e.,

$\Sigma=\{\phi \in \mathscr{M} \mid \phi$ has a multiple point or a place where its derivative vanishes or other singularities .

The space $\mathscr{M}-\Sigma$ is our space of all knots. (Remark. It is important to include in $\Sigma$ all maps which have cusp singularities, for if not every knot could be changed to the unknot by pulling it tight and, at the last moment, popping it!)

The group $\widetilde{H}^{0}(\mathscr{M}-\Sigma ; \mathbb{Q})$ contains all rational-valued knot-type invariants. (The "tilde" over $H^{0}$ indicates that we have normalized by requiring each invariant to take the value 0 on the unknot.) Clearly, $\widetilde{H}^{0}(\mathscr{M}-\Sigma ; \mathbb{Q})$ contains enough information to classify knots. For example, in principle it is possible to make a list containing all knot types, ordered, say, by crossing number, and then to delete repeats until knots which occur in distinct positions on the list have distinct knot types. The position of a knot on the list would then define an element in $\widetilde{H}^{0}(\mathscr{M}-\Sigma ; \mathbb{Q})$ which (by definition) classifies knots.

Our space $\mathscr{M}$ is much too big for direct analysis, but fortunately its very size makes it extremely flexible and subject to approximation. Let $\Gamma^{d} \subset \mathscr{M}$ be the subspace of all maps from $\mathbb{R}^{1}$ to $\mathbb{R}^{3}$ given by

$$
t \rightarrow\left(\phi_{1}(t), \phi_{2}(t), \phi_{3}(t)\right),
$$

where each $\phi_{i}$ is a polynomial of the form

$$
t^{d+1}+a_{i 1} t^{d}+\cdots+a_{i d} t
$$

with $d$ even. As $t \rightarrow \pm \infty$, the images $\phi(t)$ are seen to be asymptotic to the direction $\pm(1,1,1)$, so the images are tangent to a fixed direction at $*=\{\infty\}$. The space $\Gamma^{d}$ has three key properties:

(i) By the Weierstrass Approximation Theorem, every knot type occurs for some $\phi \in \Gamma^{d}$ for sufficiently large $d$. 
(ii) There are ways to embed $\Gamma^{d}$ in $\Gamma^{d^{\prime}}, d<d^{\prime}$. For example [V], map $\Gamma^{d} \rightarrow \Gamma^{3 d+2}$ by reparametrizing $\phi(t)$ as $\phi\left(s^{3}+s\right)$. Thus, one may choose a sequence of positive integers $d_{1}<d_{2}<d_{3}<\cdots$ so that

$$
\widetilde{H}^{0}(\mathscr{M}-\Sigma ; \mathbb{Q})=\lim _{\leftarrow} \widetilde{H}^{0}\left(\Gamma^{d_{i}}-\Gamma^{d_{i}} \cap \Sigma ; \mathbb{Q}\right) \text {. }
$$

(iii) The fact that each $\phi \in \Gamma^{d}$ is determined by $3 d$ real numbers and that each $3 d$-tuple of real numbers determines a map in $\Gamma^{d}$ allows us to identify $\Gamma^{d}$ with Euclidean space $\mathbb{R}^{3 d}$. Alexander duality then applies, and

$$
\tilde{H}^{0}\left(\Gamma^{d_{i}}-\Gamma^{d_{i}} \cap \Sigma ; \mathbb{Q}\right) \cong \bar{H}_{3 d-1}\left(\Gamma^{d_{i}} \cap \Sigma ; \mathbb{Q}\right) \text {. }
$$

where $\bar{H}_{3 d-1}$ denotes reduced homology. In other words, the object of study is no longer the topology of an individual component $\mathbf{K}$ of $\mathscr{M}-\Sigma$ but instead the topology of the walls $\Sigma$ which separate the components in our space of all knots. This change has an important consequence: it adds structure in the form of the natural stratification of $\Sigma$ which occurs via the hierarchy of its singularities.

The discriminant $\Sigma$ is of course hideously complicated, since maps in $\Sigma$ may have multiple points, tangencies, and all sorts of bad singularities; but other studies in singularity theory have shown that certain generic singularities predominate, and the next goal is to single these out. Let $\mathscr{M}_{j} \subset \Sigma$ be the subspace of maps $\phi \in \Sigma$ which have $j$ transverse double points (and possibly other singularities too). Call $\phi \in \mathscr{M}_{j}$ a j-embedding if its only singularities are $j$ transverse double points. Let $\Sigma_{j}=\left\{\phi \in \mathscr{M}_{j} / \phi\right.$ is not a $j$-embedding $\}$. Then $\Sigma \supset \Sigma_{1} \supset \Sigma_{2} \supset \cdots$. Each map in $\mathscr{M}_{j}-\Sigma_{j}$ has exactly $j$ generic singularities, where a generic singularity is a transverse double point.

The space $\mathscr{M}_{j}-\Sigma_{j}$ is not connected. Its components are singular knot types with exactly $j$ transverse double points. To illustrate how it might enter our picture, let $\mathbf{K}$ and $\mathbf{K}^{*}$ be knot types which are neighbors in $\mathscr{M}-\Sigma$. Thus a single passage across $\Sigma$ suffices to change a representative $K$ of $\mathbf{K}$ to a representative $K^{*}$ of $\mathbf{K}^{*}$. At the instant of passage across $\Sigma$ (assuming that the passage is across a "nice" part of $\Sigma$ ), one will obtain a singular knot $K^{1}$ in $\mathscr{M}_{1}$. This singular knot can be thought of as keeping track of the crossing change. The singular knot $K^{1}$ will be in $\mathscr{M}_{1}-\Sigma_{1}$. The path from $\mathbf{K}$ to $\mathbf{K}^{*}$ will in general not be unique, so it may happen that two distinct passages yield singular knots $K^{1}, N^{1}$ which are in distinct components of $\mathscr{M}_{1}-\Sigma_{1}$. To pass from one to another, one must cross $\Sigma_{1}$, obtaining (at the instant of passage) a singular knot $K^{2} \in \mathscr{M}_{2}-\Sigma_{2}$, and so forth. Vassiliev studied this situation, applying the methods of spectral sequences to obtain combinatorial conditions which determine certain families of invariants. These have become known as Vassiliev invariants. They are stable values of elements in the group $\bar{H}_{3 d-1}\left(\Gamma^{d} \cap \Sigma\right) \cong \widetilde{H}^{0}\left(\Gamma^{d}-\Gamma^{d} \cap \Sigma\right)$, as $d \rightarrow \infty$, evaluated on a component $\mathbf{K}$ of $\mathscr{M}-\Sigma$. As such, they are elements of $\tilde{H}^{0}(\mathscr{M}-\Sigma ; \mathbb{Q})$.

A Vassiliev invariant $v_{i}(\mathbf{K})$ of order $i$ takes into account information about the singular knot types near $\mathbf{K}$ in $\mathscr{M}-\Sigma, \mathscr{M}_{1}-\Sigma_{1}, \mathscr{M}_{2}-\Sigma_{2}, \ldots, \mathscr{M}_{i}-\Sigma_{i}$, where $i$ is a positive integer. The sum of two $i$ th order invariants is another invariant of order $i$. In fact, these invariants lie in a finite-dimensional vector space $V_{i}$ of dimension $d_{i}$. Clearly, these invariants have much to do with crossing changes. 
The manuscript [V] ends with a combinatorial recipe for the calculation of $v_{i}(\mathbf{K})$.

\section{AXIOMS AND INITIAL DATA FOR VASSILIEV INVARIANTS}

In 1990, when preprints of [V] were first circulated, topologists were in possession of an overflowing cornucopia of knot and link invariants. In addition to the Jones polynomial and its generalizations, we mention the knot group invariants of [Wa], the energy invariants of $[\mathrm{FH}]$, and the algebraic geometry invariants of [CCG], all of which seemed to come from mutually unrelated directions! In addition, there were generalizations of the Jones invariants to knotted graphs [Y1] and, finally, the numerical knot invariants of [V].

The first hint that the Vassiliev and Jones invariants might be related was that both extended to invariants of singular knots. A second hint was a tiny bit of evidence: the first nontrivial Vassiliev invariant is $v_{2}$, which was identified in [V] as coinciding with the second coefficient in the Conway-Alexander polynomial. In fact, that coefficient has another interpretation, as the second derivative of the Jones polynomial evaluated at 1. Studying [V] and having in mind a possible relationship with Jones invariants, the author and Lin were led to reformulate the results in [V] in terms of a set of "axioms and initial data". We describe them in this section.

Let $v: \mathscr{M}-\Sigma \rightarrow \mathbb{Q}$ denote a function from the space $\mathscr{M}-\Sigma$ of all knots to the rational numbers. This function determines a Vassiliev invariant if it satisfies the following conditions:

Axioms. To state the first axiom, we note that the singular knots in [V] are subject to an equivalence relation which is known as rigid vertex isotopy-a neighborhood of each double point of the singular knot spans an open disc $\mathbb{R}^{2} \subset$ $\mathbb{R}^{3}$-and this disc must be preserved under admissible isotopies. For example, if one begins with the standard diagram of the trefoil and of its mirror image (both shown in Figure 4) and then replaces any one crossing in each diagram by a double point, one will obtain two singular knots which are equivalent under isotopy but not under rigid vertex isotopy. This restriction is quite natural if one thinks of singular knots as objects which keep track of crossing changes in a passage from one knot to a neighbor in $\mathscr{M}-\Sigma$. Knowing that there is such a disc, or plane, it then makes sense to speak of the two resolutions (see Figure 5) at a singular point $p$ of the singular knot $K^{j}$ : We denote these two resolutions $K_{p_{+}}^{j-1}$ and $K_{p_{-}}^{j-1}$ respectively. The first axiom is a type of crossingchange formula:

$$
v\left(K_{p}^{j}\right)=v\left(K_{p_{+}}^{j-1}\right)-v\left(K_{p_{-}}^{j-1}\right) .
$$

This axiom in itself places no constraints on $v$ as a knot invariant. Rather, it serves to define $v$ on singular knots, assuming that it is known on knots.

The second axiom is what makes $v$ computable:

$$
\exists i \in Z^{+} \text {such that } v\left(\mathbf{K}^{j}\right)=0 \text { if } j>i .
$$

The smallest such $i$ is the order of $v$. To stress it, we call our invariant $v_{i}$ from now on.

Initial data. In addition to (10a) and (10b) we need initial data. The first piece of initial data relates to the normalization mentioned earlier:

$$
v_{i}(\mathbf{O})=0 \text {. }
$$




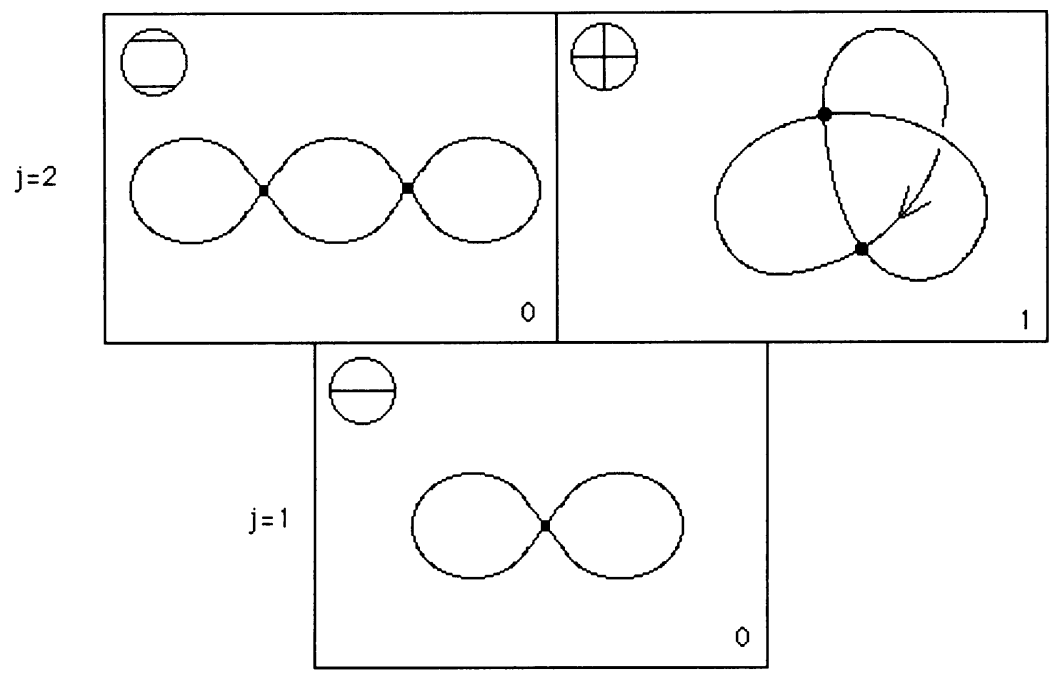

FIGURE 9. Actuality table for $i=2$.

To describe the second piece of initial data, we need a definition. A singular point on a knot diagram will be called nugatory if its positive and negative resolutions define the same knot type, in the obvious manner indicated in Figure 10. It is clear that if we are to obtain a true knot invariant, its value on $v_{i}\left(\mathbf{K}_{p_{+}}^{j-1}\right)$ and $v_{i}\left(\mathbf{K}_{p_{-}}^{j-1}\right)$ must agree when $p$ is a nugatory crossing, so by (10a) the initial data must satisfy

$$
v_{i}\left(\mathbf{K}_{p}^{j}\right)=0 \text { if } p \text { is a nugatory crossing. }
$$

The final piece of initial data is in the form of a table, but before we can describe it we need to discuss a point that we avoided earlier: The space $\mathscr{M}_{j}-\Sigma_{j}$ has a natural decomposition into components, such that two singular knots cannot define the same singular knot type if they belong to distinct components. To make this assertion precise, let $\mathbf{K}^{j}$ be a singular knot of order $j$, i.e., the image of $S^{1}$ under a $j$-embedding $\phi \in \mathscr{M}_{j}-\Sigma_{j}$. Then $\phi^{-1}\left(\mathbf{K}^{j}\right)$ is a circle with $2 j$ distinguished points, arranged in pairs, where two distinguished points are paired if they are mapped to the same double point on $\mathbf{K}^{j}$. The [j]-configuration which $\mathbf{K}^{j}$ respects is the cyclically ordered collection of point pairs. We will use a picture to define it, i.e., a circle with arcs joining the paired points, as in the top row of Figure 9, where we show the two possible [2]-configurations, together with a choice of a singular knot which respect each. The initial data must take account of the following (see [ST 1] for a proof):

Lemma 1. Two singular knots $K_{1}^{j}, K_{2}^{j}$ become equivalent after an appropriate series of crossing changes if and only if they respect the same [j]-configuration.

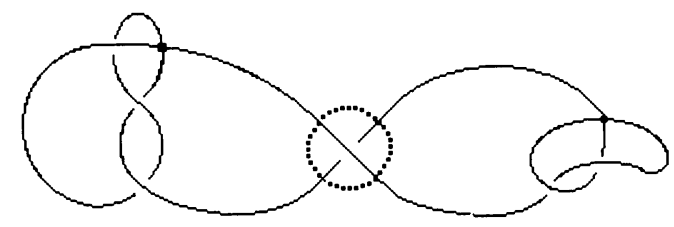

FigURE 10. Nugatory crossing. 
The table which we now construct to complete the initial data is called an actuality table. Figure 9 is an example, when $i=2$. It gives the values of $v_{i}\left(\mathbf{K}^{j}\right)$ for a representative collection of singular knots of order $j \leq i$. The table contains a choice of a singular knot $K^{j}$ which respects each $[j]$-configuration, for $j=1,2, \ldots, i$. The choice is arbitrary (however, the work in completing the table will be increased if poor choices are made). Next to each $K^{j}$ in the table is the configuration it respects, and below it is the value of $v_{i}\left(\mathbf{K}^{j}\right)$. These values are, of course, far from arbitrary, and the heart of the work in [V] is the discovery of a finite set of rules which suffice to determine them. The rules turn out to be in the form of a system of linear equations. The unknowns are the values of the functionals on the finite set of singular knots in the actuality table. The linear equations which hold between these unknowns are consequences of the local equations (which may be thought of as crossing-change formulas) illustrated in Figure 11. These equations are not difficult to understand: use (10a) to resolve each double point into a sum of two crossing points. Then each local picture in Figure 11 will be replaced by a linear combination of four pictures. The equations in Figure 11 will then be seen to reduce to a sequence of applications of Reidemeister's third move. See $\S 3$ of [BL] for a description of the method that allows one to write down the full set of equations. See $\S 2.4$ of $[\mathrm{BN}]$ for a proof that solutions to the equations, in the special case when $j=i$, may be constructed out of information about the irreducible representations of simple Lie algebras. It is not known whether the methods of [BN] yield all solutions in the case $j=i$. The extension of the solutions for the case $j=i$ to the cases $2 \leq j \leq i-1$ must be handled by the less routine methods described in [BL], at this time.

An example should suffice to illustrate that $(10 \mathrm{a})-(10 \mathrm{~d})$ and the actuality table allow one to compute $v_{i}(\mathbf{K})$ on all knots. For our example we compute $v_{2}(\mathbf{K})$ when $\mathbf{K}$ is the trefoil knot. The first picture in Figure 12 shows our
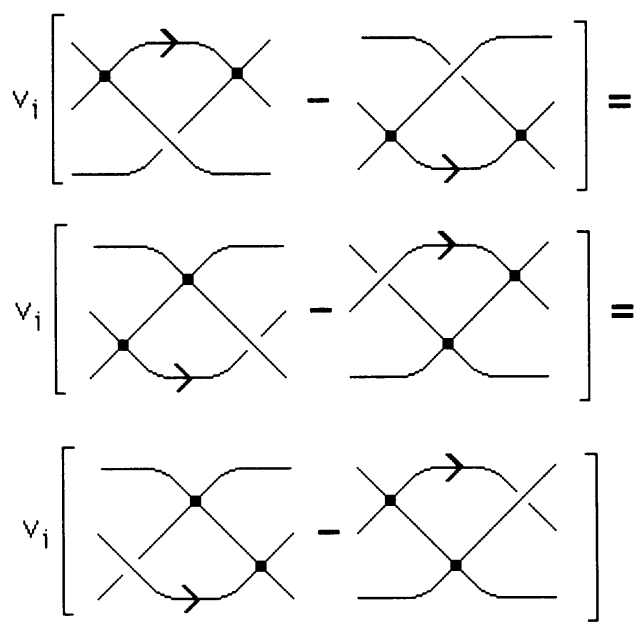

Figure 11. Crossing change formula for Vassiliev invariants of related singular knots. 


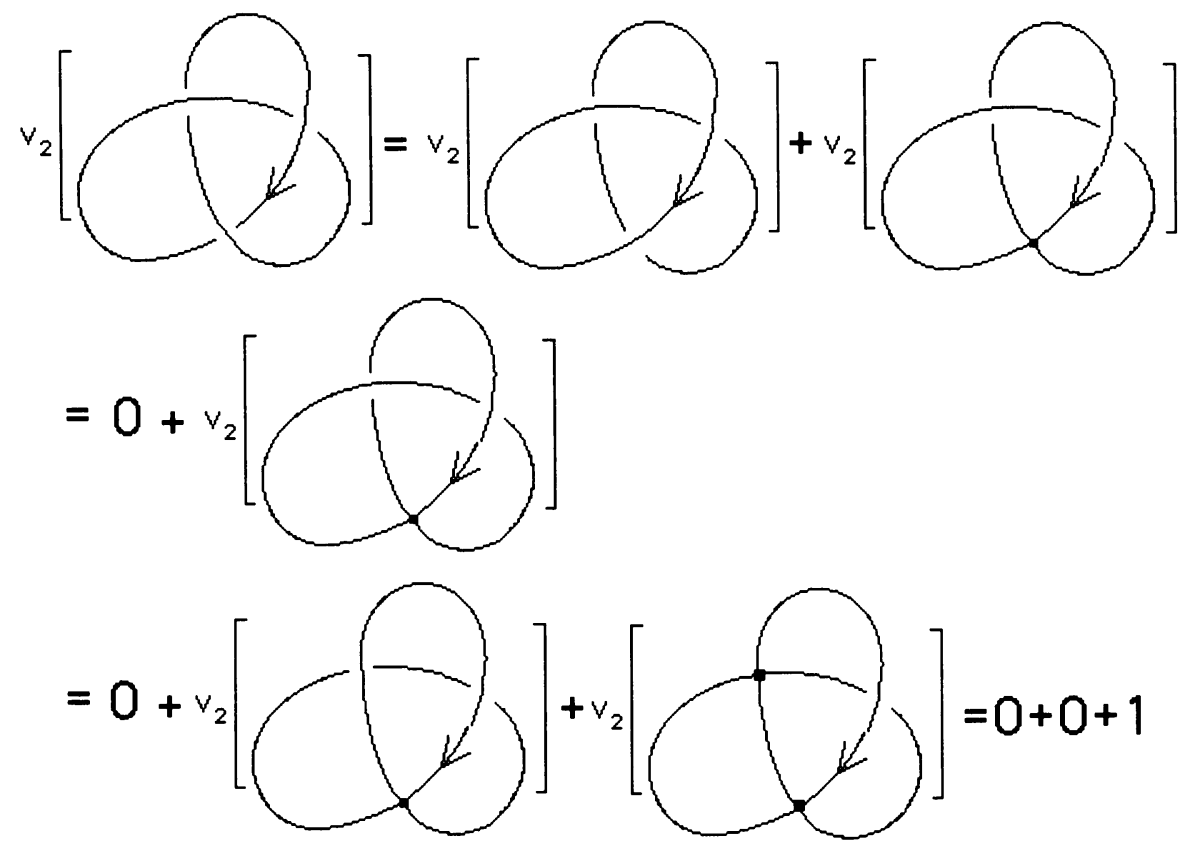

Figure 12. Computing $v_{2}(\mathbf{K})$ for the trefoil knot.

representative of the trefoil, with a crossing which is marked. Changing it, we will obtain the unknot $\mathbf{O}$.

The crossing we selected is positive and so (10a) yields

$$
v_{2}(K)=v_{2}(O)+v_{2}\left(N^{1}\right)
$$

where $N^{1}$ is the indicated singular knot. It does not have the same singular knot type as the singular knot $K^{1}$ in the table, so (using the lemma) we introduce another crossing change to modify it to the singular knot in the table which respects the unique [1]-configuration. In so doing, we obtain a singular knot $N^{2}$ with two singular points. It does not have the same singular knot type as the representative in the table which respects the same [2]-configuration, but by (10b) that does not matter. Thus, using (10b), the calculation comes to an end in finitely many steps.

\section{SINGULAR BRAIDS}

In $\S 3$ we showed that any generalized Jones invariant may be obtained from a Markov trace on an appropriate family of finite-dimensional matrix representations of the braid groups. Up to now, braids have not entered the picture as regards Vassiliev invariants, but that is easy to rectify. To do so, we need to extend the usual notions of braids and closed braids to singular braids and closed singular braids.

A representative $K^{j}$ of a singular knot or link $\mathbf{K}^{j}$ will be said to be a closed singular braid if there is an axis $A$ in $\mathbb{R}^{3}$ (think of it as the $z$-axis) such that if $K^{j}$ is parametrized by cylindrical coordinates $(z, \theta)$ relative to $A$, the polar angle function restricted to $K^{j}$ is monotonic increasing. This implies that $K^{j}$ meets each half-plane $\theta=\theta_{0}$ in exactly $n$ points, for some $n$. In [Al1] Alexander proved the well-known fact that every knot or link $\mathbf{K}$ may be 
so represented, and we begin our work by extending his theorem to singular knots and links.

Lemma 2. Let $K^{j}$ be an arbitrary representative of a singular knot or link $\mathbf{K}^{j}$. Choose any copy $A$ of $\mathbb{R}^{1}$ in $\mathbb{R}^{3}-K^{j}$. Then $K^{j}$ may be deformed to a closed singular $n$-braid, for some $n$, with $A$ as axis.

Proof. Regard $A$ as the $z$-axis in $\mathbb{R}^{3}$. After an isotopy of $K^{j}$ in $\mathbb{R}^{3}-A$ we may assume that $K^{j}$ is defined by a diagram in the $(r, \theta)$-plane. By a further isotopy we may also arrange that each singular point $p_{k}$ has a neighborhood $N\left(p_{k}\right) \in K^{j}$ such that the polar angle function restricted to $\bigcup_{k=1}^{j} N\left(p_{k}\right)$ is monotonic increasing. The proof then proceeds exactly as in [Al1], vis: Modify $K^{j}-\bigcup_{k=1}^{j} N\left(p_{k}\right)$ to a piecewise linear family of arcs $\mathscr{A}$, subdividing the collection if necessary so that each $\alpha \in \mathscr{A}$ contains at most one undercrossing or overcrossing of the knot diagram. After a small isotopy we may assume that the polar angle function is nonconstant on each $\alpha \in \mathscr{A}$. Call an arc $\alpha \in \mathscr{A}$ bad or good accordingly as the polar angle function is increasing or decreasing on $\alpha$. If there are no bad arcs, we will have a closed braid, so we may assume there is at least one, say $\beta$. Modify $K^{j}$ by replacing $\beta$ by two good edges $\beta_{1} \cup \beta_{2}$ as in Figure 13. The only possible obstruction is if the interior of the triangle which is bounded by $\beta \cup \beta_{1} \cup \beta_{2}$ is pierced by the rest of $K^{j}$, but that may always be avoided by choosing the new vertex $\beta_{1} \cap \beta_{2}$ so that it lies very far above (resp. below) the rest of $K^{j}$ if the arc $\beta$ contains an under(resp. over-) crossing of the diagram. Induction on the number of bad edges completes the proof.

In view of Lemma 2, every singular knot in the actuality table of $\S 5$ may be chosen to be a closed singular braid. To continue, we split these closed singular braids open along a plane $\theta=\theta_{0}$ to "open" singular braids, which we now define. In $\S 3$ we described a geometric braid as a pattern of $n$ interwoven strings in $\mathbb{R}^{2} \times I \subset \mathbb{R}^{3}$ which join $n$ points, labeled $1,2, \ldots, n$ in $\mathbb{R}^{2} \times\{0\}$ to the corresponding points in $\mathbb{R}^{2} \times\{1\}$, intersecting each intermediate plane $\mathbb{R}^{2} \times\{t\}$ in exactly $n$ points. To extend to singular braids, it is only necessary to weaken the last condition to allow finitely many values of $t$ at which the braid meets $\mathbb{R}^{2} \times\{t\}$ in $n-1$ points instead of $n$ points. Two singular braids are equivalent if they are isotopic through a sequence of singular braids, the isotopy fixing the initial and end points of each singular braid strand. Singular braids compose like ordinary braids: concatenate two patterns, erase the middle plane, and rescale.

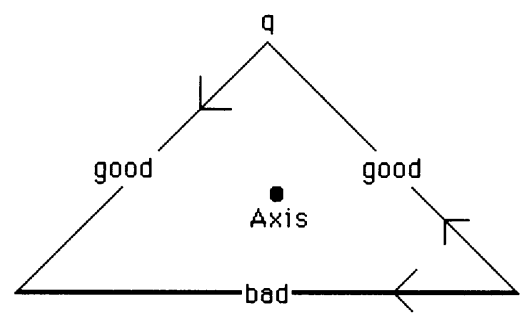

FIGURE 13. Replacing a bad arc by two good arcs. 
Choose any representative of an element of $S B_{n}$. After an isotopy we may assume that distinct double points occur at distinct $t$-levels. From this it follows that $S B_{n}$ is generated by the elementary braids $\sigma_{1}, \ldots, \sigma_{n-1}$ and the elementary singular braids $\tau_{1}, \ldots, \tau_{n-1}$ of Figure 8 . We distinguish between the $\sigma_{i}$ 's and the $\tau_{i}$ 's by calling them crossing points and double points respectively in the singular braid diagram. Both determine double points in the projection.

The manuscript [Bae] lists defining relations in $S B_{n}$ as:

$$
\begin{gathered}
{\left[\sigma_{i}, \sigma_{j}\right]=\left[\sigma_{i}, \tau_{j}\right]=\left[\tau_{i}, \tau_{j}\right]=0 \text { if }|i-j| \geq 2,} \\
{\left[\sigma_{i}, \tau_{i}\right]=0,} \\
\sigma_{i} \sigma_{j} \sigma_{i}=\sigma_{j} \sigma_{i} \sigma_{j} \quad \text { if }|i-j|=1, \\
\sigma_{i} \sigma_{j} \tau_{i}=\tau_{j} \sigma_{i} \sigma_{j} \quad \text { if }|i-j|=1,
\end{gathered}
$$

where in all cases $1 \leq i, j \leq n-1$. The same set of relations also occurs in $[\mathrm{KV}]$ as generalized Reidemeister moves. The validity of these relations is easily established via pictures; for example, see Figure 14 for special cases of (11a)(11d). To the best of our knowledge, however, there is not even a sketch of a proof that they suffice in the literature, so we sketch one now, as it will be important for us that no additional relations are needed.

Lemma 3. The monoid $S B_{n}$ is generated by $\left\{\sigma_{i}, \tau_{i} ; 1 \leq i \leq n-1\right\}$. Defining relations are (11a)-(11d).

Proof. We have already indicated a proof that the $\sigma_{i}$ 's and the $\tau_{i}$ 's generate $S B_{n}$, so the only question is whether every relation is a consequence of (11a)(11d).

We regard our braids as being defined by diagrams. Let $\bar{z}, \bar{z}^{\prime}$ be singular braids which represent the same element of $S B_{n}$, and let $\left\{\bar{z}_{s} ; s \in I\right\}$ denote the family of singular braids which join them. The fact that the intermediate braid diagrams $\bar{z}_{s}$ have no triple points implies that there is a well-defined

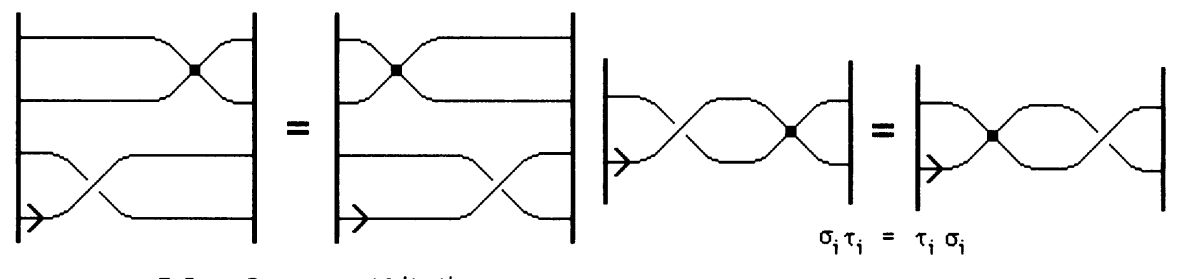

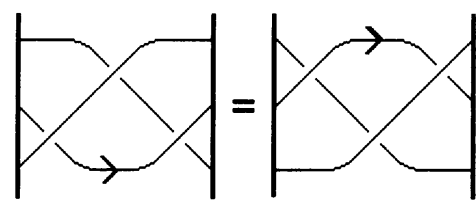

$\sigma_{i} \sigma_{j} \sigma_{i}=\sigma_{j} \sigma_{i} \sigma_{j} \quad$ if $|i-j|=1$

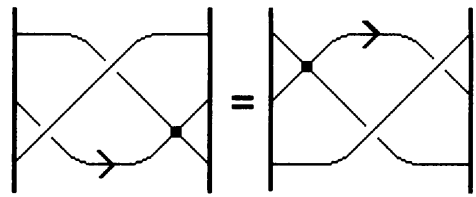

$\sigma_{i} \sigma_{j} \tau_{i}=\tau_{j} \sigma_{i} \sigma_{j} \quad$ if $|i-j|=1$

FIGURE 14. Relations in $S B_{n}$. 
order of the singularities along each braid strand which is preserved during the isotopy, and this allows us to set up a 1-1 correspondence between double points in $\bar{z}$ and $\bar{z}^{\prime}$. We now examine the other changes which occur during the isotopy. Divide the $s$-interval $[0,1]$ into small subintervals, during which exactly one of the following changes occurs in the sequence of braid diagrams:

(i) Two double points in the braid projection interchange their $t$-levels. See relations (11a) and (11b) and Figure 14.

(ii) A triple point in the projection is created momentarily as a "free" strand crosses a double point or a crossing point in the projection. See (11d) and Figure 14.

(iii) New crossing points in the knot diagram are created or destroyed. See Reidemeister's second move in Figure 2.

All possible cases of (i) are described by relations (11a) and (11b). Noting that the $\sigma_{i}$ 's are invertible and that the mirror image of $\sigma_{i}$ (resp. $\left.\sigma_{i}^{-1}, \tau_{i}\right)$ is $\sigma_{i}^{-1}$ (resp. $\left.\sigma_{i}, \tau_{i}\right)$, it is a simple exercise to see that consequences of $(11 \mathrm{c})$ and (11d) cover all possible cases of (ii). As for (iii), if we restrict to small $s$-intervals about the instant of creation or destruction, these will occur in pairs and be described by the trivial relation $\sigma_{i} \sigma_{i}^{-1}=\sigma_{i}^{-1} \sigma_{i}=1$. Outside of these special $s$-intervals the singular braid diagram will be modified by isotopy, which contributes no new relations. Thus relations (11a)-(11d) are defining relations for $S B_{n}$.

Now something really interesting happens. Let $\tilde{\sigma}_{i}$ denote the image of the elementary braid $\sigma_{i}$ under the natural map from the braid group $B_{n}$ to its group algebra $\mathbb{C} B_{n}$.

Theorem 2 (cf. [Bae, Lemma 1]). The map $\eta: S B_{n} \rightarrow \mathbb{C} B_{n}$ which is defined by $\eta\left(\sigma_{i}\right)=\tilde{\sigma}_{i}, \eta\left(\tau_{i}\right)=\tilde{\sigma}_{i}-\tilde{\sigma}_{i}^{-1}$ is a monoid homomorphism.

Proof. Check to see that relations (11a)-(11d) are consequences of the braid relations in $\mathbb{C} B_{n}$.

Corollary 1. Every finite-dimensional matrix representation $\rho_{n}: B_{n} \rightarrow \mathrm{GL}_{n}(\mathscr{E})$ extends to a representation $\tilde{\rho}_{n}$ of $S B_{n}$, defined by setting $\tilde{\rho}_{n}\left(\tau_{i}\right)=\rho_{n}\left(\sigma_{i}\right)-$ $\rho_{n}\left(\sigma_{i}^{-1}\right)$.

Proof. Clear.

Thus, in particular, all R-matrix representations of $B_{n}$ extend to representations of the singular braid monoid $S B_{n}$.

Remark 1. Recall that in Figure 11 we gave picture examples of some of the relations which need to be satisfied by a functional on the knot space in order for the indices in an actuality table to determine a knot type invariant. Unlike the relations which we depicted in Figure 14, the ones in Figure 11 are, initially, somewhat mysterious. However, if one passes via Theorem 2 to the group algebra $\mathbb{C} B_{n}$ of the braid group, replacing each $\tau_{i}$ by $\sigma_{i}-\sigma_{i}^{-1}$, it will be seen that these relations actually hold in the algebra, not simply in the space of VBLfunctionals. This fact is additional evidence of the naturality of the map $\eta$ of Theorem 2 and indeed of the Vassiliev construction. We conjecture that the kernel of $\eta$ is trivial, i.e., that a nontrivial singular braid in the monoid $S B_{n}$ never maps to zero in the group algebra $\mathbb{C} B_{n}$. 
Remark 2. Various investigators, for example, Kauffman in [Kau1], have considered a somewhat different monoid which we shall call the tangle monoid, obtained by adding the "elementary tangle" $\varepsilon_{i}$ of Figure 8 to the braid group. The tangle monoid, however, does not map homomorphically to $\mathbb{C} B_{n}$, and one must pass to quotients of $\mathbb{C} B_{n}$, for example, the so-called Birman-Wenzl algebra [BW, We1], to give it algebraic meaning. In that sense the tangle monoid appears to be less fundamental than the singular braid monoid.

\section{The Proof of Theorem 1}

We are now ready to prove Theorem 1 . We refer the reader to the introduction for its statement.

Theorem 1 was first proved by the author and Lin in [BL] for the special cases of the HOMFLY and Kauffman polynomials and then in full generality for all quantum group invariants in [L]. The proof we give here is new. It is modeled on the proof for the special cases in [BL]. We like it because it is simple and because it gives us an opportunity to show that the braid groups, which are central to the study of the Jones invariants, are equally useful in Vassiliev's setting. The tools in our proof are the R-matrix representations of $\S 3$, the axioms and initial data of $\S 5$, and the singular braid monoid of $\S 6$. Theorem 1 also is implicit in [Bae], which was written simultaneously with and independently of this manuscript. The techniques used there are very similar to ours, but the goal is different.

Proof of Theorem 1. By hypothesis, we are given a quantum group invariant $\mathscr{J}_{q}: \mathscr{M}-\Sigma \rightarrow \mathscr{E}$, where $\mathscr{E}$ denotes a ring of Laurent polynomials over the integers in powers of $q$ (or in certain cases powers of roots of $q$ ). Using Lemma 2 , we find a closed braid representative $K_{\beta}$ of $\mathbf{K}, \beta \in B_{n}$. We then pass to the R-matrix representation $\rho_{n, R}: B_{n} \rightarrow \mathrm{GL}_{m_{n}}(\mathscr{E})$ associated to $\mathscr{J}_{q}$. By Corollary 1 that representation extends to a representation $\tilde{\rho}_{n, R}$ of $S B_{n}$. By equation (9) the Laurent polynomial $\mathscr{J}_{q}(\mathbf{K})$ is the trace of $\rho_{n, R}(\beta) \cdot \mu$, where $\mu$ is the enhancement of $\rho_{n, R}$.

As was discussed in $\S 3$, our representation $\rho_{n, R}$ is determined by the choice of a matrix $R$ which acts on the vector space $V \otimes 2$. Lin notes in Lemma 1.3 of [L] that on setting $q=1$ the matrix $R=\left(R_{i_{1} i_{2}}^{j_{1} j_{2}}\right)$ goes over to $\mathrm{id}_{V} \otimes \mathrm{id}_{V}$. From this it follows that $\rho_{n, R}\left(\sigma_{i}\right)$ (which acts on $V^{\otimes n}$ ) has order 2 at $q=1$. Hence, we conclude that $\rho_{n, R}$ goes over to a representation of the symmetric group if we set $q=1$, with $\sigma_{i}$ going to the transposition $(i, i+1)$. In particular, this means that at $q=1$ the images under $\rho_{n, R}$ of $\sigma_{i}$ and $\sigma_{i}^{-1}$ will be identical, which, in turn, means that the images of $\sigma_{i}$ and $\sigma_{i}^{-1}$ under $\rho_{n, R}$ coincide at $q=1$.

Armed with this knowledge, we change variables, as in the statement of Theorem 1, replacing $q$ by $e^{x}$. Expanding the powers of $e^{x}$ in its Taylor series, the image of an arbitrary element $\beta$ under $\rho_{n, R}$ will be a matrix power series

$$
\rho_{n, R}(\beta)=M_{0}(\beta)+M_{1}(\beta)+M_{2}(\beta)+\cdots
$$

where each $M_{i}(\beta) \in \mathrm{GL}_{m_{n}}(Q)$.

Lemma 5 (cf. [Bae, Corollary 1]). In the extended representation, $M_{0}\left(\tau_{i}\right)=0$.

Proof. Since $M_{0}\left(\sigma_{i}\right)=M_{0}\left(\sigma_{i}^{-1}\right)$, the assertion follows. 
Now let us turn our attention to the power series expansion of $\mathscr{J}_{q}$, i.e., to $\mathscr{J}_{x}(\mathbf{K})=\sum_{i=0}^{\infty} u_{i}(\mathbf{K}) x^{i}$. The coefficients $u_{i}(\mathbf{K})$ in this series, as $\mathbf{K}$ ranges over all knot types, determine a functional $u_{i}: \mathscr{M}-\Sigma \rightarrow Q$. We wish to prove that $u_{i}$ is a Vassiliev invariant of order $i$.

By $\S 5$ it suffices to prove that, if we use (10a) to extend the definition of $u_{i}$ to singular knots, then (10b)-(10d) will be satisfied and a consistent actuality table exists. The first thing to notice is that, since we began with a knot-type invariant $\mathscr{J}_{q}$, the functional $u_{i}$ is also a knot-type invariant. From this it follows that its extension to singular knots is also well defined, so using our knowledge of $u_{i}$ on knots we can fill in the actuality table.

The second observation is that $(10 \mathrm{c})$ is satisfied, because every generalized Jones invariant satisfies $\mathscr{J}_{q}(\mathbf{O}) \equiv 1$, and from this it follows that $\mathscr{P}_{x}(\mathbf{O}) \equiv 1$. As for (10d), it is also satisfied, for if not $u_{i}$ could not be a knot-type invariant. Thus the only problem which remains is to prove that $u_{i}$ satisfies axiom (10b). However, notice that by Lemma 5 we have

$$
\tilde{\rho}_{n, R}\left(\tau_{i}\right)=M_{1}\left(\tau_{i}\right) x+M_{2}\left(\tau_{i}\right) x^{2}+M_{3}\left(\tau_{i}\right) x^{3}+\cdots,
$$

and from this it follows that if $\mathbf{K}^{j}$ is a singular knot which has $j$ singular points, then any singular closed braid $K_{\gamma}^{j}, \gamma \in S B_{r}$, which represents $\mathbf{K}^{j}$ will also have $j$ singularities. The singular braid word $\gamma$ will then contain $j$ elementary singular braids. From this it follows that

$$
\tilde{\rho}_{n, R}(\gamma)=M_{j}(\gamma) x^{j}+M_{j+1}(\gamma) x^{j+1}+\cdots .
$$

The coefficient of $x^{i}$ in this power series is $u_{i}\left(\mathbf{K}^{j}\right)$. But then, $u_{i}\left(\mathbf{K}^{j}\right)=0$ if $i<j$, i.e., axiom (10b) is also satisfied and the proof of Theorem 1 is complete.

\section{OPEN PROBLEMS}

Our story is almost ended, so it is appropriate to recapitulate and ask what we have gained by our interpretation of the quantum group invariants as Vassiliev invariants? The goal has been to bring topology into the picture, and indeed we have done so because we have shown that, when the Jones invariant of a knot $\mathbf{K}$ is expanded in a power series in $x$, the coefficient of $x^{i}$ gives information about certain stable homology groups of the discriminant $\Sigma$, in a neighborhood of $\mathbf{K}$. The information concerns its structure at "depth" $i$; that is, of course, only a beginning. The discriminant $\Sigma$ is a complicated subset of an infinitedimensional space, $\mathscr{M}$, and there seems to be no way to begin to visualize $\Sigma$. Indeed, the work has just begun.

The study of Vassiliev invariants is fairly new. We now review some of the things that have been learned about them during the past few years. A natural place to start is with the special cases when $i$ is small, and this was already done in [V]. The invariant $v_{1}(\mathbf{K})$ is zero for all knots $\mathbf{K}$. The first nontrivial Vassiliev invariant has order 2, and there is a one-dimensional vector space of such invariants. However, $v_{2}(\mathbf{K})$ was well known to topologists in other guises before either Jones or Vassiliev came on the scene, vis:

(i) It is the second coefficient in Conway's version of the Alexander polynomial [C].

(ii) Its mod 2 reduction is the Arf invariant of a knot, which has to do with cobordism. 
It now has other interpretations too:

(iii) It is the "total twist" of a knot, as defined in [LM]. It may be computed from the following recursive formula:

$$
v_{2}\left(K_{p_{+}}\right)-v_{2}\left(K_{p_{-}}\right)=\mathscr{L} k\left(K_{p_{0}}\right)
$$

where $\mathscr{L} k$ denotes the linking number of the two-component link $K_{p_{0}}$.

(iv) It is the second derivative of the Jones polynomial $J_{q}(\mathbf{K})$, evaluated at $q=1$.

Unfortunately, all of these facts do not allow us to understand the significance of $v_{2}$ for the topology of $\Sigma$. There is much work to be done. As for $v_{3}(\mathbf{K})$, it also belongs to a one-dimensional vector space of invariants, but to the best of our knowledge no one has yet identified it as a classical invariant-indeed, nothing much seems to be known about it.

With regard to the higher-order invariants, recall that Vassiliev invariants of order $i$ belong to a linear vector space $V_{i}$. This space is the space of $i$ th order invariants modulo those of order $i-1$. The first characteristic of this vector space to question is its dimension $m_{i}$, for $i>2$. This seems to be a deep and difficult combinatorial problem for arbitrary $i$, and at this writing the best we can do is to compute, the issue being the construction of the actuality table for an invariant of order $i$. This problem divides naturally into two parts: the first is to determine the Vassiliev invariants of the singular knots $K^{i}$ in the top row. By axiom (10b) they only depend upon the [i]-configuration which they respect, so their determination is easier than the corresponding problem for the remaining rows. The former problem is solved by setting up and solving the system of linear equations (3.11) of [BL]. The dimension of the space of solutions is the sought-for integer $m_{i}$. One year ago Bar Natan wrote a computer program which listed the distinct [i]-configurations and calculated the dimensions (top row only) for $i \leq 7$. Very recently Stanford developed a different computer program which checked that all of Bar Natan's "top row" solutions, for $i \leq 7$, extend to the remaining rows of the actuality table, i.e., to solutions to the somewhat more complicated set of equations (3.17) of [BL], so Bar Natan's numbers are actually the dimensions $m_{i}$ that we seek. The results of the two calculations are:

$\begin{array}{cccccccc}i & 1 & 2 & 3 & 4 & 5 & 6 & 7 \\ m_{i} & 0 & 1 & 1 & 3 & 4 & 9 & 14\end{array}$

The data we just gave leads us to an important question. We proved, in Theorem 1, that

$$
\{\text { Quantum group invariants }\} \subseteq\{\text { Vassiliev invariants }\} .
$$

The question is: Is the inclusion proper? The data is relevant because it was thought that the question might be answered by showing that for some fixed $i$ the quantum group invariants spanned a vector space of dimension $d_{i}<m_{i}$; however, a dimension count to $i=6$ shows that there are enough linearly independent invariants coming from quantum groups to span the vector spaces $V_{i}$. Bar Natan's calculations showed that $d_{7}$ is at least 12 , whereas $m_{7}=14$; 
however, the data on the quantum group invariants is imprecise because the invariants which come from the nonexceptional Lie algebras begin to make their presence felt as $i$ increases. The only one of those which has been investigated, to date, is $G_{2}$.

For $i=8$ the computations themselves create difficulties. Bar Natan's calculation is close to the edge of what one can do, because to determine $m_{8}$ he found he had to solve a linear system consisting of 334,908 equations in 41,874 unknowns (the number of distinct [8]-configurations with no separating arcs). His approximate calculation shows that the solution space is of dimension 27. However, even if his answer is correct, we would still need to compute the rest of the actuality table before we could be sure that $m_{8}=27$ rather than $m_{8} \leq 27$.

Vassiliev invariants actually form an algebra, not simply a sequence of vector spaces, because the product of a Vassiliev invariant of order $p$ and one of $q$ is a Vassiliev invariant of order $p+q$. This was proved by Lin (unpublished), using straightforward methods, and more indirectly by Bar Natan [BN]. Thus the dimension $\hat{m}_{i}$ of new invariants is in general smaller than $m_{i}$, because the data in our table includes invariants which are products of ones of smaller order. It is a simple matter to correct the given data to find $\hat{m}_{i}$. For example, an invariant of order 4 could be the product of two invariants of order 2 ; so when we correct for the fact that there is a one-dimensional space of invariants of order 2 , we see that $\hat{m}_{4}=3-1=2$. For $i=1,2,3,4,5,6,7$ we find that $\hat{m}_{i}=0,1,1,2,3,5,8$, i.e., the beginning of the Fibbonaci sequence! This caused some excitement until Bar Natan's computation of $m_{8}$ showed that $\hat{m}_{8}$ was at most 12 , not 13 . The asymptotic behavior of $m_{i}$ as $i \rightarrow \infty$ is a very interesting problem indeed.

We can approach the question of whether the inclusion is proper from a different point of view. One of the earliest problems in knot theory concerned the fundamental symmetries which are always present in the definition of knot type. We defined knot type to be the topological equivalence class of the pair $\left(S^{3}, K\right)$ under homeomorphisms which preserve the given orientation on both $S^{3}$ and $K$. A knot type is called amphicheiral if it is equivalent to the knot type obtained by reversing the orientation of $S^{3}$ (but not $K$ ) and invertible if it is equivalent to the knot type obtained by reversing the orientation on $K$ (but not $S^{3}$ ). As noted earlier, Max Dehn proved in 1913 that nonamphicheiral knots exist [De], but remarkably, it took over forty years before it became known that noninvertible knots exist [Tr]. The relevance of this matter to our question is: While the quantum group invariants detect nonamphicheirality of knots, they do not detect noninvertibility of knots. So, if we could prove that Vassiliev invariants distinguished a single noninvertible knot from its inverse, the answer to our question, Is the inclusion proper? would be yes. We know we cannot answer the question this way for $i \leq 7$, and as noted above $i=8$ presents serious computational difficulties. On the other hand, the theoretical problem seems to be unexpectedly subtle. Thus, at this moment, the matter of whether Vassiliev invariants ever detect noninvertibility remains open.

Setting aside empirical evidence and unsolved problems, we can ask some easy questions which will allow us to sharpen the question of whether Jones or Vassiliev invariants determine knot type. As was noted in $\S 2$, there are three very intuitive invariants which have, to date, proved to be elusive: the crossing number $c(\mathbf{K})$, the unknotting number $u(\mathbf{K})$, and the braid index $s(\mathbf{K})$. Clearly 
these determine functionals on the space $\mathscr{M}-\Sigma$, and so they determine elements in the group $\widetilde{H}^{0}(\mathscr{M}-\Sigma)$. Vassiliev invariants lie in a sequence of approximations to $\widetilde{H}^{0}(\mathscr{M}-\Sigma)$. So a reasonable question to ask is, Are $c(\mathbf{K}), u(\mathbf{K})$, and $s(\mathbf{K})$ Vassiliev invariants? Theorem 5.1 of [BL] shows that $u(\mathbf{K})$ is not, and the proof given there is easily modified to show that $c(\mathbf{K})$ and $s(\mathbf{K})$ are not either. So, at the very least, we have learned that there are integer-valued functionals on Vassiliev's space of all knots which are not Vassiliev invariants. This leaves open the question of whether there are sequences of Vassiliev invariants which converge to these invariants.

Another question which has been asked is, How powerful are the Vassiliev invariants, if we restrict our attention to invariants of bounded order? The answer to that question is, not very good, based on examples which were discovered, simultaneously and independently, by Lin [Li2] and by Stanford [St2]. We now describe Stanford's construction, which is particularly interesting from our point of view because it is based on the closed-braid approach to knots and links. See Remark (ii) for a description of Lin's construction.

To state his theorem, we return to braids. Let $P_{n}$ be the pure braid group, i.e., the kernel of the natural homomorphism from $B_{n}$ to the symmetric group $S_{n}$. The groups of the lower central series $\left\{P_{n}^{k} ; k=1,2, \ldots\right\}$ of $P_{n}$ are defined inductively by $P_{n}^{1}=P_{n}, P_{n}^{k}=\left[P_{n}, P_{n}^{k-1}\right]$. Notice that if $\beta \in B_{n}$, with the closed braid $\widehat{\beta}$ a knot, then $\widehat{\alpha \beta}$ will also be a knot for every $\alpha \in P_{n}$.

Theorem 3 [St2]. Let $\mathbf{K}$ be any knot type, and let $K_{\beta}$, where $\beta \in B_{n}$, be any closed braid representative of $\mathbf{K}$. Choose any $\alpha \in P_{n}^{k}$. Then the Vassiliev invariants of order $\leq k$ of the knots $K_{\beta}$ and $K_{\alpha \beta}$ coincide.

Remarks. (i) Using the results in [BM1], Stanford has constructed sequences $\alpha_{1}, \alpha_{2}, \ldots$ of 3 -braids such that the knot types obtained via Theorem 3 are all distinct and prime. Intuition suggests that distinct $\alpha_{j}$ 's will always give distinct knot types, but at this writing that has not been proved.

(ii) One may choose $K_{\beta}$ in Theorem 3 to represent the unknot and obtain infinitely many distinct knots all of whose Vassiliev invariants of order $\leq k$, for any $k$, are zero. Lin's construction gives other such examples. In particular, he proves that, if $K$ is any knot and if $K(m)$ is its $m$ th iterated (untwisted) Whitehead double, then all Vassiliev invariants of order $\leq(m+1)$ of $K(m)$ are zero. This allows him to construct infinitely many composite knots which have the same properties as Stanford's knots. It is not clear whether his construction is a special case of Stanford's construction.

(iii) The special case of the unknot and the one-variable Jones polynomial is interesting. Theorem 3 says that if we expand the Jones polynomials of the knots in the collection in power series and truncate those series by cutting off all terms of $x$-degree bigger than $k$, the result will be zero. This is far from saying that the polynomials themselves are trivial. Indeed, the question of whether there is a nontrivial knot which has the same one-variable Jones polynomial as the unknot remains an open problem and an intriguing one. Note that our lack of knowledge about this problem is in striking contrast to the control mathematicians now have over the Alexander polynomial: understanding its topological meaning, we also know precisely how to construct knots with Alexander polynomial 1 . 
(iv) For a brief period (before a mistake was discovered in the proof) it seemed as if there might be an affirmative answer to the following question: Given any two distinct knots $\mathbf{K}$ and $\mathbf{K}^{*}$, does there exist a sequence of Vassiliev invariants $\left\{w_{i} \mid i=1,2,3, \ldots\right\}$ and an integer $N$ such that $w_{i}(\mathbf{K}) \neq$ $w_{i}\left(\mathbf{K}^{*}\right) \forall i \geq N$ ? Notice that even if the answer was yes, it would not solve the knot problem, because if we had explicit examples $\mathbf{K}$ and $\mathbf{K}^{*}$ and if we knew the sequence, we still would not know how large $N$ had to be. So after letting the computer run all weekend without a definitive answer, we would not know whether the knots were really the same or whether we simply had given up too soon. However, this is probably the very best that one could hope for from the algebraic invariants.

(v) By contrast to all of this, the work of Haken [Ha] and the work of Hemion $[\mathrm{He}]$ show that there is an algorithm which distinguishes knots. In recent years efforts have been directed at making that algorithm workable (for example, see [JT] for a discussion of recent results), but much work remains to be done before it could be considered "practical", even for the simplest examples.

(vi) The work in [BM2] and related papers referenced therein is aimed at a different algorithm which is based upon the theory of braids. At present it has resulted in a very rapid hand calculation for definitively distinguishing knots and links which are defined by closed braids with at most 3 strands, with partial results for the general case.

(vii) Among the special cases for which an effective classification scheme exists we mention (in addition to the work just cited on links defined by closed 3-braids) the cases of links with 2-bridges and of algebraic (in the sense of algebraic geometry) links. For more information on these and other classical topics, see [Rol] and [BZ].

(viii) An extensive guide to the "pre-Jones" literature may be found in [BZ].

\section{ACKNOWLEDGMENTS}

Many people helped us to understand this subject. We single out, especially: V. I. Arnold (who first stimulated our interest in Vassiliev invariants), XiaoSong Lin, and Dror Bar Natan. Discussions with Vaughan Jones, Vladimir Turaev, Michael Polyak, Alex Sossinsky, and Ted Stanford have also been very helpful to us. We also thank Oleg Viro for telling us about the parallel theory of knot invariants which was developed by $\mathrm{M}$. Gusarov [Gu].

\section{REFERENCES}

[Al1] J. W. Alexander, A lemma on systems of knotted curves, Proc. Nat. Acad. Sci. U.S.A. 9 (1923), 93-95.

[A12] J. W. Alexander, Topological invariants of knots and links, Trans. Amer. Math. Soc. 30 (1928), 275-306.

[Arn1] V. I. Arnold (ed.), Theory of singularities and its applications, Adv. in Soviet Math., vol. 1, Amer. Math. Soc., Providence, RI, 1990.

[Arn2] The cohomology ring of the colored braid group, Notes Acad. Sci. USSR 5 (1969), 227-232.

[Art] E. Artin, Theorie der Zöpfe, Hamburg Abh. 4 (1925), 47-72.

[Bae] J. Baez, Link invariants of finite type and perturbation theory, Lett. Math. Phys. (to appear). 
[Bax] R. J. Baxter, Exactly solvable models in statistical mechanics, Academic Press, London, 1982.

[BD] A. A. Belavin and V. G. Drinfeld, On classical Yang-Baxter equation for simple Lie algebras, Funct. Anal. Appl. 16 (1982), 1-10.

[Be] D. Bennequin, Entrlacements et équations de Pfaffe, Asterisque 107-108 (1983), 87-161.

[Bi] J. S. Birman, Braids, links and mapping class groups, Ann. of Math Stud., vol. 84, Princeton Univ. Press, Princeton, NJ, 1974.

[BL] J. S. Birman and X. S. Lin, Knot polynomials and Vassiliev invariants, Invent. Math (to appear).

[BM1] J. S. Birman and W. W. Menasco, Studying links via closed braids III: Classifying links which are closed 3-braids, Pacific J. Math. (to appear).

[BM2] Studying links via closed braids VI: A non-finiteness theorem, Pacific J. Math., vol. 156 (1992), 265-285.

[BN] D. Bar Natan, On the Vassiliev knot invariants, Harvard Univ., preprint 1992.

[BW] J. S. Birman and H. Wenzl, Braids, link polynomials and a new algebra, Trans. Amer. Math. Soc. 313 (1989), 249-273.

[BZ] G. Burde and H. Zieschang, Knots, de Gruyter, Berlin and New York, 1985.

[C] J. H. Conway, An enumeration of knots and links and some of their algebraic properties, Computational Problems in Abstract Algebra (J. Leech, ed.), Pergamon Press, New York, 1970, pp. 329-358.

[CCG] D. Cooper, M. Culler, H. Gillet, D. D. Long, and P. B. Shalen, Plane curves associated to character varieties of 3-manifolds, Univ. of California, Santa Barbara, preprint 1991.

[CGL] M. Culler, C. McA. Gordon, J. Leucke and P. B. Shalen, Dehn surgery on knots, Ann. of Math. (2) 125 (1987), 237-300.

[De] M. Dehn, Die beiden Kleeblattschlingen, Math. Ann. 75 (1914), 402-413.

[Dr1] V. G. Drinfeld, On a constant quasi-classical solution to the quantum Yang-Baxter equation, Doklady Acad. Nauk SSSR 273 (1983), 531-550.

[Dr2] - Quantum groups, Proc. ICM Berkeley, vol. 1, Amer. Math. Soc., Providence, RI, 1987, pp. 798-820.

[FH] M. Freedman and Z. H. He, On the energy of knots and unknots, Univ. of California, San Diego, preprint 1992.

[FHL] P. Freyd, J. Hoste, W. B. R. Lickorish, K. Millett, A. Ocneanu, and D. Yetter, A new polynomial invariant of knots and links, Bull. Amer. Math. Soc. 12 (1985), 239-246.

[FW] J. Franks and R. F. Williams, Braids and the Jones polynomial, Trans. Amer. Math. Soc. 303 (1987), 97-108.

[GL] C. McA. Gordon and J. Leucke, Knots are determined by their complements, Bull. Amer. Math. Soc. 20 (1989), 83-88.

[Go] L. Goeritz, Bermerkungen zur knotentheorie, Hamburg Abh. 10 (1934), 201-210.

[Gu] M. Gusarov, A new form of the Conway-Jones polynomial for knots via von Neumann algebras, Zap. Nauchn. Sem. Leningrad Otdel. Mat. Inst. Steklov. (LOMI) 193 (1991), 4-9.

[Ha] W. Haken, Über das Homömorphieproblem der 3-Mannigfaltigkeiten, Math. Z. 80 (1962), 89-120.

[He] G. Hemion, On the classification of homeomorphisms of 2-manifolds and the classification of 3-manifolds, Acta Math. 142 (1979), 123-155.

[Ho] J. Hoste, A polynomial invariant of knots and links, Pacific J. Math. 124 (1986), 295-320.

[Je] L. Jeffrey, Chern-Simons-Witten invariants of lens spaces and torus bundles, and the semiclassical approximation, Oxford Univ., preprint 1992.

[Ji1] M. Jimbo, A q-difference analogue of $U(g)$ and the Yang-Baxter equation, Lett. Math. Phys. 10 (1985), 63-69.

[Ji2] _ Quantum R-matrix for the generalized Toda system, Comm. Math. Physics 102 (1986), 537-547. 
[Jo1] V. F. R. Jones, Index for subfactors, Invent. Math. 72 (1983), 1-25.

[Jo2] _ Braid groups, Hecke algebras and type $\mathrm{II}_{1}$ factors, Geometric Methods in Operator Theory, Pitman Res. Notes Math. Ser., vol. 123, Longman Sci. Tech., Harlow, 1986, pp. 242-273.

[Jo3] - A polynomial invariant for knots via von Neumann algebras, Bull. Amer. Math. Soc. 12 (1985), 103-111.

[Jo4] Hecke algebra representations of braid groups and link polynomials, Ann. of Math. (2) 126 (1987), 335-388.

[Jo5] - On knot invariants related to some statistical mechanics models, Pacific J. Math. 137 (1989), 311-334.

[JR] V. Jones and M. Rosso, Invariants of torus knots derived from quantum groups, Abstracts Amer. Math. Soc., Abstract no. 874-16-124 (1992).

[JT] W. Jaco and J. L. Tollefson, Algorithms for the complete decomposition of a closed 3manifold, Univ. of Conn. at Storres, preprint 1992.

[Kal] E. Kalfagianni, The $G_{2}$ link invariant, Columbia Univ., preprint 1992.

[Kan] T. Kanenobu, Infinitely many knots with the same polynomial, Proc. Amer. Math. Soc. 97 (1986), 158-162.

[Kau 1] L. H. Kauffman, An invariant of regular isotopy, Trans. Amer. Math. Soc. 318 (1990), 417-471.

[Kau 2] - States models and the Jones polynomial, Topology 26 (1987), 395-407.

[KM] R. Kirby and P. Melvin, The 3-manifold invariants of Witten and Reshetikhin-Turaev for $S L(2, \mathbb{C})$, Invent. Math. 105 (1991), 473-545.

[Koh1] T. Kohno, Monodromy representations of braid groups and Yang-Baxter equations, Ann. Inst. Fourier (Grenoble) 37 (1987), 139-160.

[Koh 2] Linear representations of braid groups and classical Yang-Baxter equations, Contemp. Math., vol. 78, Amer. Math. Soc., Providence, RI, 1988, pp. 339-363.

[Kon] M. Kontsevich, Vassiliev's knot invariants, Adv. in Soviet Math. (to appear).

[Ku] G. Kuperberg, The 1, 0, 1, 1, 4, 10 Ansatz, preprint.

[KV] L. Kauffman and P. Vogel, Link polynomials and a graphical calculus, J. Knot Theory Ramifications 1 (1992), 59-104.

[La] R. Lawrence, Homological representations of the Hecke algebras, Comm. Math. Phys. 135 (1990), 141-191.

[Li1] X. S. Lin, Vertex models, quantum groups and Vassiliev's knot invariants, Columbia Univ., preprint 1991.

[Li2] _ Finite type link invariants of 3-manifolds, Columbia Univ., preprint 1992.

[LM] W. B. R. Lickorish and K. Millett, A polynomial invariant for knots and links, Topology 26 (1987), 107-141.

[Mak] G. S. Makanin, On an analogue of the Alexander-Markov theorem, Math. USSR Izv. 34 (1990), 201-211.

[Mar] A. A. Markov, Über die freie Äquivalenz geschlossener Zöpfe, Recueil Math. Moscou 1 (1935), 73-78.

[Me] W. W. Menasco, A proof of the Bennequin-Milnor unknotting conjecture, SUNY at Buffalo, preprint 1992.

[Mo1] H. Morton, Threading knot diagrams, Math. Proc. Cambridge Philos. Soc. 99 (1986), 247-260.

[Mo2] , Seifert circles and knot polynomials, Math. Proc. Cambridge Philos. Soc. 99 (1986), 107-109.

[Mu] K. Murasugi, The Jones polynomial and classical conjectures in knot theory, Topology 26 (1987), 187-194.

[Pi] S. Piunikhin, Weights of Feynman diagrams and Vassiliev knot invariants, Moscow State Univ., preprint 1992.

[Pr] C. Procesi, The invariant theory of $n$ by $n$ matrices, Adv. in Math. 19 (1976), 306-381. 
[PT] J. Przytycki and P. Traczyk, Conway algebras and skein equivalence of links, Proc. Amer. Math. Soc. 100 (1987), 744-748.

[Re1] N. Yu. Reshetikhin, Quasitriangular Hopf algebras and invariants of tangles, Leningrad Math. J. 1 (1990), 491-513.

[Re2] - Quantized universal enveloping algebras, the Yang-Baxter equation and invariants of links, LOMI preprints E-4-87, E-17-87 (1988).

[Rol] D. Rolfsen, Knots and links, Publish or Perish, Berkeley, CA, 1976.

[Ros] M. Rosso, Groupes quantique et modèles à vertex de V. Jones en théorie des noeuds, C. R. Acad. Sci. Paris Sér. I Math. 307 (1988), 207-210.

[RT] N. Reshetikhin and V. G. Turaev, Invariants of 3-manifolds via link polynomials and quantum groups, Invent. Math. 103 (1991), 547-597.

[Sei] H. Seifert, Verschlingungsinvarianten, Sber. Preuss. Akad. Wiss. 26 (1933), 811-828.

[Sem] M. Semenov Tian-Shansky, What is a classical R-matrix?, Funct. Anal. Appl. 17 (1983), 259-270.

[Si] J. Simon, How many knots have the same group?, Proc. Amer. Math. Soc. 80 (1980), 162-166.

[St1] T. Stanford, Finite type invariants of knots, links and graphs, Columbia Univ., preprint 1992.

[St2] _ Braid commutators and Vassiliev invariants, Columbia Univ., preprint 1992.

[Ta] P. G. Tait, On knots I, II and III, Scientific Papers of P. G. Tait, vol. 1, Cambridge Univ. Press, Cambridge and New York, 1988, pp. 273-347.

[Tr] H. Trotter, Non invertible knots exist, Topology 2 (1964), 275-280.

[Tu] V. Turaev, The Yang-Baxter equation and invariants of links, Invent. Math. 92 (1988), 527-553.

[V1] V. A. Vassiliev, Cohomology of knot spaces, Theory of Singularities and its Applications (V. I. Arnold, ed.), Amer. Math. Soc., Providence, RI, 1990, pp. 23-69.

[V2] Topology of complements to discriminants and loop spaces, Theory of Singularities and its Applications (V. I. Arnold, ed.), Amer. Math. Soc., Providence, RI, 1990, pp. 9-21.

[Wa] M. Wada, Group invariants of links, Topology 31 (1992), 399-406.

[WAD] M. Wadati, Y. Akutsu, and T. Deguchi, Link polynomials and exactly solvable models, Non-Linear Physics (Gu, Li, and Tu, eds.), Springer-Verlag, Berlin, 1990.

[We1] H. Wenzl, Quantum groups and subfactors of type B, C and D, Comm. Math. Phys. 133 (1990), 383-432.

[We2] Representations of braid groups and the quantum Yang-Baxter equation, Pacific $\mathbf{J}$. Math. 145 (1990), 153-180.

[Wi] E. Witten, Quantum field theory and the Jones polynomial, Comm. Math. Phys. 121 (1989), 351-399.

[Wh] W. Whitten, Knot complements and groups, Topology 26 (1987), 41-44.

[WX] A Weinstein and P. Xu, Classical solutions of the quantum Yang-Baxter equation, Comm. Math. Phys. 148 (1992), 309-343.

[Y1] S. Yamada, An invariant of spacial graphs, J. Graph Theory 13 (1989), 537-551.

[Y2] - The minimum number of Seifert circles equals the braid index, Invent. Math. 89 (1987), 346-356.

Department of Mathematics, Columbia University, New York, New York 10027

E-mail address: jb@math.columbia.edu 